\title{
CENTRAL LOCAL DISCONTINUOUS GALERKIN METHODS ON OVERLAPPING CELLS FOR DIFFUSION EQUATIONS
}

\author{
Yinguie Liu ${ }^{1}$, Chi-Wang Shu ${ }^{2}$, Eitan Tadmor ${ }^{3}$ and Mengping Zhang ${ }^{4}$
}

\begin{abstract}
In this paper we present two versions of the central local discontinuous Galerkin (LDG) method on overlapping cells for solving diffusion equations, and provide their stability analysis and error estimates for the linear heat equation. A comparison between the traditional LDG method on a single mesh and the two versions of the central LDG method on overlapping cells is also made. Numerical experiments are provided to validate the quantitative conclusions from the analysis and to support conclusions for general polynomial degrees.
\end{abstract}

Mathematics Subject Classification. 65M60.

Received December 9, 2009. Revised January 6, 2011.

Published online June 13, 2011.

\section{INTRODUCTION}

In this paper we present two versions of the central local discontinuous Galerkin (LDG) methods on overlapping cells for solving diffusion equations, and provide their stability analysis and error estimates for the linear heat equation. We also compare the traditional LDG method on a single mesh and the two versions of the central LDG methods on overlapping cells in this context.

The LDG method is designed by suitably rewriting the diffusion partial differential equation (PDE) as a first-order system and then discretizing it by the DG method [3]. For a general review of DG methods we refer to [4]. In this paper we use overlapping cells and hence duplicative information, thereby avoiding numerical fluxes which is a distinct advantage of central schemes. This work is a continuation of our earlier work in [6,7] in which we presented and analyzed central DG schemes for hyperbolic PDEs. We would like to point out that the discussion on the difficulties related to numerical fluxes for DG methods solving diffusion equations has

\footnotetext{
Keywords and phrases. Central discontinuous Galerkin method, local discontinuous Galerkin method, overlapping cells, diffusion equation, heat equation, stability, error estimate.

${ }^{1}$ School of Mathematics, Georgia Institute of Technology, Atlanta, 30332-0160 GA, USA. yingjie@math.gatech.edu

Research supported in part by NSF grant DMS-0810913.

2 Division of Applied Mathematics, Brown University, Providence, RI 02912, USA. shu@dam.brown.edu Research supported in part by DOE grant DE-FG02-08ER25863 and NSF grant DMS-0809086.

${ }^{3}$ Department of Mathematics, Institute for Physical Science and Technology and Center of Scientific Computation and Mathematical Modeling (CSCAMM), University of Maryland, College Park, MD 20742, USA. tadmor@cscamm.umd.edu Research supported in part by NSF grants DMS-1008397, FRG-0757227 and ONR grant N00014-09-10385.

${ }^{4}$ Department of Mathematics, University of Science and Technology of China, Hefei, Anhui 230026, P.R. China. mpzhang@ustc.edu.cn

Research supported in part by NSFC grant 11071234 and CAS grant KJCX1-YW-21.
} 
attracted a lot of attention in the literature, for example we mention the recovery type DG method by van Leer and Nomura [8] (see also [9]), and the hybridizable discontinuous Galerkin (HDG) method of Cockburn et al. [5].

Our overall goal is to design (central) LDG methods which serve as effective solvers for convection diffusion equations, ranging from scalar equations of the form

$$
u_{t}+f(u)_{x}=\left(c(u) u_{x}\right)_{x}, \quad c(u) \geq 0
$$

to general multidimensional systems of equations. The purely convective case was studied by us in [6,7]. The main focus of this paper is therefore to initiate the study of central LDG methods for the diffusive case, where we limit ourselves to a modest beginning with the linear heat equation

$$
u_{t}=u_{x x}, \quad(x, t) \in[a, b] \times[0, T] .
$$

The formulation of the LDG and central LDG methods for the heat equation (1.2), can be extended in a straightforward manner to general nonlinear convection diffusion equations, see [3,6]. However, the stability and convergence analysis is more subtle: our stability analysis and error estimates are restricted to the heat equation with periodic boundary conditions and can be generalized to non-periodic boundary conditions, based on the finite element techniques. Stability analysis of the (central) LDG method for more general nonlinear diffusion is left for a future work.

The starting point of the LDG method is to rewrite the heat equation (1.2) as a first-order system

$$
u_{t}-r_{x}=0, \quad r-u_{x}=0
$$

Let $\left\{x_{j}\right\}$ be a partition of $[a, b]$ with $h_{j+\frac{1}{2}}=x_{j+1}-x_{j}$ and $h=\max _{j} h_{j+\frac{1}{2}}$. The mesh is regular, in the sense that $\max _{j} h_{j+\frac{1}{2}} / \min _{j} h_{j+\frac{1}{2}}$ is upper-bounded by a fixed constant during mesh refinements. Denote $x_{j+\frac{1}{2}}=$ $\frac{1}{2}\left(x_{j+1}+x_{j}\right), I_{j}=\left(x_{j-\frac{1}{2}}, x_{j+\frac{1}{2}}\right)$, and $I_{j+\frac{1}{2}}=\left(x_{j}, x_{j+1}\right)$. $V_{h}$ is the set of piecewise polynomials of degree $k$ over the subintervals $\left\{I_{j}\right\}$ with no continuity assumed across the subinterval boundaries. Likewise, $W_{h}$ is the set of piecewise polynomials of degree $k$ over the subintervals $\left\{I_{j+\frac{1}{2}}\right\}$ with no continuity assumed across the subinterval boundaries.

The traditional LDG method is defined by formally using the DG method in equations (1.3). The semidiscrete version is as follows. Find $u_{h}(\cdot, t) \in V_{h}$ and $r_{h}(\cdot, t) \in V_{h}$, such that for any $\varphi_{h} \in V_{h}$ and $\psi_{h} \in V_{h}$,

$$
\begin{gathered}
\int_{I_{j}} \partial_{t} u_{h} \varphi_{h} \mathrm{~d} x=-\int_{I_{j}} r_{h} \partial_{x} \varphi_{h} \mathrm{~d} x+\hat{r}_{j+\frac{1}{2}} \varphi_{h}\left(x_{j+\frac{1}{2}}^{-}\right)-\hat{r}_{j-\frac{1}{2}} \varphi_{h}\left(x_{j-\frac{1}{2}}^{+}\right) \\
\int_{I_{j}} r_{h} \psi_{h} \mathrm{~d} x=-\int_{I_{j}} u_{h} \partial_{x} \psi_{h} \mathrm{~d} x+\hat{u}_{j+\frac{1}{2}} \psi_{h}\left(x_{j+\frac{1}{2}}^{-}\right)-\hat{u}_{j-\frac{1}{2}} \psi_{h}\left(x_{j-\frac{1}{2}}^{+}\right) .
\end{gathered}
$$

Here $\varphi_{h}\left(x_{j+\frac{1}{2}}^{ \pm}\right)$refers to the right and left limits, respectively, of the possibly discontinuous function $\varphi_{h}(x)$ at the cell interface $x_{j+\frac{1}{2}}$. A good choice for the numerical fluxes (the hat terms), both in accuracy and in compactness of the eventual stencil after the auxiliary variable $r_{h}$ is eliminated, is

$$
\hat{u}_{j+\frac{1}{2}}=u_{h}\left(x_{j+\frac{1}{2}}^{+}, t\right), \quad \hat{r}_{j+\frac{1}{2}}=r_{h}\left(x_{j+\frac{1}{2}}^{-}, t\right)
$$

i.e. we alternatively take the left and right limits for the fluxes in $u$ and $r$ (we could of course also take the pair $u_{h}\left(x_{j+\frac{1}{2}}^{-}, t\right)$ and $r_{h}\left(x_{j+\frac{1}{2}}^{+}, t\right)$ as the fluxes $)$.

The first version of central LDG method. On overlapping cells uses both the spaces $V_{h}$ and $W_{h}$. The first semi-discrete version has the following form. Find $u_{h}(\cdot, t), r_{h}(\cdot, t) \in V_{h}$ and $v_{h}(\cdot, t), s_{h}(\cdot, t) \in W_{h}$, such that 
for any $\varphi_{h}, \bar{\varphi}_{h} \in V_{h}$ and $\psi_{h}, \bar{\psi}_{h} \in W_{h}$, we have

$$
\begin{aligned}
\int_{I_{j}} \partial_{t} u_{h} \varphi_{h} \mathrm{~d} x & =-\int_{I_{j}} s_{h} \partial_{x} \varphi_{h} \mathrm{~d} x+s_{h}\left(x_{j+\frac{1}{2}}, t\right) \varphi_{h}\left(x_{j+\frac{1}{2}}^{-}\right)-s_{h}\left(x_{j-\frac{1}{2}}, t\right) \varphi_{h}\left(x_{j-\frac{1}{2}}^{+}\right) \\
\int_{I_{j}} r_{h} \bar{\varphi}_{h} \mathrm{~d} x & =-\int_{I_{j}} v_{h} \partial_{x} \bar{\varphi}_{h} \mathrm{~d} x+v_{h}\left(x_{j+\frac{1}{2}}, t\right) \bar{\varphi}_{h}\left(x_{j+\frac{1}{2}}^{-}\right)-v_{h}\left(x_{j-\frac{1}{2}}, t\right) \bar{\varphi}_{h}\left(x_{j-\frac{1}{2}}^{+}\right) \\
\int_{I_{j+\frac{1}{2}}} \partial_{t} v_{h} \psi_{h} \mathrm{~d} x & =-\int_{I_{j+\frac{1}{2}}} r_{h} \partial_{x} \psi_{h} \mathrm{~d} x+r_{h}\left(x_{j+1}, t\right) \psi_{h}\left(x_{j+1}^{-}\right)-r_{h}\left(x_{j}, t\right) \\
\psi_{h}\left(x_{j}^{+}\right) \int_{I_{j+\frac{1}{2}}} s_{h} \bar{\psi}_{h} \mathrm{~d} x & =-\int_{I_{j+\frac{1}{2}}} u_{h} \partial_{x} \bar{\psi}_{h} \mathrm{~d} x+u_{h}\left(x_{j+1}, t\right) \bar{\psi}_{h}\left(x_{j+1}^{-}\right)-u_{h}\left(x_{j}, t\right) \bar{\psi}_{h}\left(x_{j}^{+}\right) .
\end{aligned}
$$

The second version of the central LDG method. Has a similar form to the first one: the only difference from (1.7)-(1.10) is the addition of an extra term involving the difference between the two duplicative solutions on different cells. This term serves as an additional numerical dissipation and we will see later that the addition of this term allows us to recover optimal convergence rate for the piecewise linear case, as well as providing smaller errors for non-smooth initial conditions. The semi-discrete version is as follows. Find $u_{h}(\cdot, t), r_{h}(\cdot, t) \in V_{h}$ and $v_{h}(\cdot, t), s_{h}(\cdot, t) \in W_{h}$, such that for any $\varphi_{h}, \bar{\varphi}_{h} \in V_{h}$ and $\psi_{h}, \bar{\psi}_{h} \in W_{h}$, we have

$$
\begin{aligned}
\int_{I_{j}} \partial_{t} u_{h} \varphi_{h} \mathrm{~d} x= & \frac{1}{\tau_{\max }} \int_{I_{j}}\left(v_{h}-u_{h}\right) \varphi_{h} \mathrm{~d} x-\int_{I_{j}} s_{h} \partial_{x} \varphi_{h} \mathrm{~d} x \\
& +s_{h}\left(x_{j+\frac{1}{2}}, t\right) \varphi_{h}\left(x_{j+\frac{1}{2}}^{-}\right)-s_{h}\left(x_{j-\frac{1}{2}}, t\right) \varphi_{h}\left(x_{j-\frac{1}{2}}^{+}\right) \\
\int_{I_{j}} r_{h} \bar{\varphi}_{h} \mathrm{~d} x= & -\int_{I_{j}} v_{h} \partial_{x} \bar{\varphi}_{h} \mathrm{~d} x \\
& +v_{h}\left(x_{j+\frac{1}{2}}, t\right) \bar{\varphi}_{h}\left(x_{j+\frac{1}{2}}^{-}\right)-v_{h}\left(x_{j-\frac{1}{2}}, t\right) \bar{\varphi}_{h}\left(x_{j-\frac{1}{2}}^{+}\right) \\
\int_{I_{j+\frac{1}{2}}} \partial_{t} v_{h} \psi_{h} \mathrm{~d} x= & \frac{1}{\tau_{\max }} \int_{I_{j+\frac{1}{2}}}\left(u_{h}-v_{h}\right) \psi_{h} \mathrm{~d} x-\int_{I_{j+\frac{1}{2}}} r_{h} \partial_{x} \psi_{h} \mathrm{~d} x \\
& +r_{h}\left(x_{j+1}, t\right) \psi_{h}\left(x_{j+1}^{-}\right)-r_{h}\left(x_{j}, t\right) \psi_{h}\left(x_{j}^{+}\right) \\
\int_{I_{j+\frac{1}{2}}} s_{h} \bar{\psi}_{h} \mathrm{~d} x= & -\int_{I_{j+\frac{1}{2}}} u_{h} \partial_{x} \bar{\psi}_{h} \mathrm{~d} x \\
& +u_{h}\left(x_{j+1}, t\right) \bar{\psi}_{h}\left(x_{j+1}^{-}\right)-u_{h}\left(x_{j}, t\right) \bar{\psi}_{h}\left(x_{j}^{+}\right)
\end{aligned}
$$

where $\tau_{\max }$ is an upper bound of the time step size due to the CFL restriction, that is, $\tau_{\max }=c h^{2}$ with a given constant CFL number $c$ dictated by stability.

Both versions of the central LDG methods on overlapping cells do not need a numerical flux to define the interface values of the solution, since the evaluation of the solution at the interface is in the middle of the staggered mesh, hence in the continuous region of the solution. The initial condition is taken as the $L^{2}$ projection of the PDE initial condition into the relevant finite element space.

The organization of the paper is as follows. In Section 2 we analyze the stability and give an error estimate for the first version of the central LDG scheme (1.7)-(1.10) on overlapping cells. We will see that only a sub-optimal $O\left(h^{k}\right)$ error estimate can be proved for piecewise polynomials of degree $k$. This sub-optimal convergence rate 
is also verified by numerical experiments to be sharp for $k=1$. In Section 3 we analyze the stability and give an error estimate for the second version of the central LDG scheme (1.11)-(1.14) on overlapping cells. We still can only get the sub-optimal $O\left(h^{k}\right)$ error estimate using the finite element technique, however a Fourier analysis indicates the optimal $O\left(h^{k+1}\right)$ convergence rate for $k=0$ and $k=1$ and this optimal rate is confirmed numerically together with the cases with higher $k$ in Section 4 . A comparison among the traditional LDG scheme and the two versions of central LDG schemes on overlapping cells is also given in Section 4 . Concluding remarks are provided in Section 5 .

\section{AnAlysis of the First Version of the CENTRAL LDG SCHEME ON OVERLAPPing CELLS}

We study the $L^{2}$ stability of the LDG scheme on overlapping cells (1.7)-(1.10) for the equation (1.2) in Section 2.1. In Section 2.2 we provide an $L^{2}$ a priori error estimate for smooth solutions. In Section 2.3 we give a quantitative error estimate for this central LDG scheme for the polynomial degree up to 1 using Fourier analysis, similar to the technique used in $[7,10,11]$. As indicated before, we assume periodic boundary conditions.

\section{1. $L^{2}$ stability}

Theorem 2.1. The numerical solution $u_{h}, r_{h}, v_{h}$ and $s_{h}$ of the LDG scheme (1.7)-(1.10) for the equation (1.2) satisfies the following $L^{2}$ stability condition

$$
\frac{1}{2} \frac{\mathrm{d}}{\mathrm{d} t} \int_{a}^{b}\left(\left(u_{h}\right)^{2}+\left(v_{h}\right)^{2}\right) \mathrm{d} x+\int_{a}^{b}\left(r_{h}^{2}+s_{h}^{2}\right) \mathrm{d} x=0
$$

Proof. Taking the test functions $\varphi_{h}=u_{h}$ and $\bar{\psi}_{h}=s_{h}$ in (1.7) and (1.10) respectively, summing up over $j$, and observing the periodic boundary condition, we have

$$
\begin{aligned}
& \frac{1}{2} \frac{\mathrm{d}}{\mathrm{d} t} \int_{a}^{b} u_{h}^{2} \mathrm{~d} x+\int_{a}^{b}\left(s_{h}\right)^{2} \mathrm{~d} x \\
= & -\sum_{j}\left[\int_{x_{j-\frac{1}{2}}}^{x_{j}} \partial_{x}\left(u_{h} s_{h}\right) \mathrm{d} x+\int_{x_{j}}^{x_{j+\frac{1}{2}}} \partial_{x}\left(u_{h} s_{h}\right) \mathrm{d} x-s_{h}\left(x_{j+\frac{1}{2}}, t\right) u_{h}\left(x_{j+\frac{1}{2}}^{-}, t\right)\right. \\
& \left.+s_{h}\left(x_{j-\frac{1}{2}}, t\right) u_{h}\left(x_{j-\frac{1}{2}}^{+}, t\right)-u_{h}\left(x_{j+1}, t\right) s_{h}\left(x_{j+1}^{-}, t\right)+u_{h}\left(x_{j}, t\right) s_{h}\left(x_{j}^{+}, t\right)\right] \\
= & 0 .
\end{aligned}
$$

Similarly, taking the test functions $\bar{\varphi}_{h}=r_{h}$ and $\psi_{h}=v_{h}$ in (1.8) and (1.9) respectively, we have

$$
\frac{1}{2} \frac{\mathrm{d}}{\mathrm{d} t} \int_{a}^{b} v_{h}^{2} \mathrm{~d} x+\int_{a}^{b} r_{h}^{2} \mathrm{~d} x=0
$$

Summing up the two equalities proves the theorem.

Remark 2.1. The proof of Theorem 2.1 is similar to the proof of the cell entropy inequality for the traditional LDG method in [3]. However, we cannot prove a similar $L^{2}$ stability result for the central LDG scheme on overlapping cells when applied to the nonlinear diffusion equation (1.1), even though the scheme itself can be easily defined for such nonlinear problems. The proof of Theorem 2.1 can be easily generalized to multidimensional central LDG schemes on overlapping cells for the linear diffusion equation. 


\section{2. $L^{2}$ a priori error estimate}

In this subsection we use the standard DG techniques [3] to obtain an a priori $L^{2}$ error estimates for the first version of the central LDG scheme on overlapping cells given in (1.7)-(1.10).

Theorem 2.2. The numerical solution $u_{h}, r_{h}, v_{h}$ and $s_{h}$ of the central LDG scheme on overlapping cells (1.7)(1.10) for the equation (1.2) with a smooth initial condition $u(\cdot, 0) \in H^{k+2}$ satisfies the following $L^{2}$ error estimate

$$
\left\|u-u_{h}\right\|^{2}+\left\|u-v_{h}\right\|^{2}+\left\|\left.\left|u_{x}-r_{h}\right|\right|^{2}+\right\|\left|u_{x}-s_{h}\right| \|^{2} \leq C h^{2 k}
$$

where $u$ is the exact solution of (1.2), $k$ is the polynomial degree in the finite element spaces $V_{h}$ and $W_{h}$, and the constant $C$ depends on the $(k+2)$-th order Sobolev norm of the initial condition $\|u(\cdot, 0)\|_{H^{k+2}}$ as well as on the final time $t$, but is independent of the mesh size $h$. The unmarked norm $\|\cdot\|$ here and below is the standard $L^{2}$ norm. The norm $\|\cdot\| \cdot \|$ is defined as

$$
\|\mid r(\cdot, t)\|^{2}=\int_{0}^{t}\|r(\cdot, \tau)\|^{2} \mathrm{~d} \tau .
$$

Proof. Let us first introduce the shorthand notation

$$
\begin{aligned}
& B_{j}\left(u_{h}, s_{h} ; \varphi_{h}, \bar{\psi}_{h}\right) \\
= & \int_{I_{j}} \partial_{t} u_{h} \varphi_{h} \mathrm{~d} x+\int_{I_{j}} s_{h} \partial_{x} \varphi_{h} \mathrm{~d} x-s_{h}\left(x_{j+\frac{1}{2}}, t\right) \varphi_{h}\left(x_{j+\frac{1}{2}}^{-}\right)+s_{h}\left(x_{j-\frac{1}{2}}, t\right) \varphi_{h}\left(x_{j-\frac{1}{2}}^{+}\right) \\
& +\int_{I_{j+\frac{1}{2}}} s_{h} \bar{\psi}_{h} \mathrm{~d} x+\int_{I_{j+\frac{1}{2}}} u_{h} \partial_{x} \bar{\psi}_{h} \mathrm{~d} x-u_{h}\left(x_{j+1}, t\right) \bar{\psi}_{h}\left(x_{j+1}^{-}\right)+u_{h}\left(x_{j}, t\right) \bar{\psi}_{h}\left(x_{j}^{+}\right) .
\end{aligned}
$$

By the definition of the scheme, we have:

$$
B_{j}\left(u_{h}, s_{h} ; \varphi_{h}, \bar{\psi}_{h}\right)=0
$$

for all $j$ and all $\varphi_{h} \in V_{h}$ and $\bar{\psi}_{h} \in W_{h}$. It can also be easily verified that the exact solutions $u$ and $r=u_{x}$ of the PDE (1.2) satisfy

$$
B_{j}\left(u, r ; \varphi_{h}, \bar{\psi}_{h}\right)=0
$$

for all $j$ and all $\varphi_{h} \in V_{h}$ and $\bar{\psi}_{h} \in W_{h}$. This is usually referred to as the consistency of the scheme. Subtracting (2.4) from (2.5), we obtain the error equation which represents the Galerkin orthogonality

$$
B_{j}\left(u-u_{h}, r-s_{h} ; \varphi_{h}, \bar{\psi}_{h}\right)=0
$$

for all $j$ and all $\varphi_{h} \in V_{h}$ and $\bar{\psi}_{h} \in W_{h}$.

We now define $P$ and $Q$ as the standard $L^{2}$ projection into $V_{h}$ and $W_{h}$ respectively. That is, for each $j$,

$$
\int_{I_{j}}(P w(x)-w(x)) \varphi_{h}(x) \mathrm{d} x=0 \quad \forall \varphi_{h} \in \mathbb{P}^{k}\left(I_{j}\right)
$$

and

$$
\int_{I_{j+\frac{1}{2}}}(Q w(x)-w(x)) \psi_{h}(x) \mathrm{d} x=0 \quad \forall \psi_{h} \in \mathbb{P}^{k}\left(I_{j+\frac{1}{2}}\right)
$$

where $\mathbb{P}^{k}\left(I_{j}\right)$ and $\mathbb{P}^{k}\left(I_{j+\frac{1}{2}}\right)$ denote the spaces of polynomials of the degree up to $k$ in the cell $I_{j}$ and the cell $I_{j+\frac{1}{2}}$ respectively. Standard approximation theory [2] implies, for a smooth function $w$,

$$
\|(P w(x)-w(x))\|+h^{1 / 2}\|(P w(x)-w(x))\|_{\Gamma_{j}} \leq C h^{k+1}
$$


and

$$
\|(Q w(x)-w(x))\|+h^{1 / 2}\|(Q w(x)-w(x))\|_{\Gamma_{j+\frac{1}{2}}} \leq C h^{k+1}
$$

where $\Gamma_{j}$ and $\Gamma_{j+\frac{1}{2}}$ denote the set of boundary points of all elements $I_{j}$ and $I_{j+1 / 2}$ respectively, and the positive constant $C$, here and below, solely depending on $w(x)$ and its first $k+2$ derivatives, is independent of $h$.

We also recall that [2], for any $w_{h} \in V_{h}$ or $w_{h} \in W_{h}$, there exists a positive constant $C$ independent of $w_{h}$ and $h$, such that

$$
\left\|\partial_{x} w_{h}\right\| \leq C h^{-1}\left\|w_{h}\right\| ; \quad\left\|w_{h}\right\|_{\Gamma} \leq C h^{-1 / 2}\left\|w_{h}\right\|
$$

where $\Gamma=\Gamma_{j}$ or $\Gamma_{j+\frac{1}{2}}$.

We now take:

$$
\varphi_{h}=P u-u_{h}, \quad \bar{\psi}_{h}=Q r-s_{h}
$$

in the error equation (2.6), and denote

$$
\varphi^{e}=P u-u, \quad \psi^{e}=Q r-r
$$

to obtain

$$
B_{j}\left(\varphi_{h}, \bar{\psi}_{h} ; \varphi_{h}, \bar{\psi}_{h}\right)=B_{j}\left(\varphi^{e}, \psi^{e} ; \varphi_{h}, \bar{\psi}_{h}\right) .
$$

For the left-hand side of (2.14), we use a similar proof as that for Theorem 2.1 to conclude

$$
\sum_{j} B_{j}\left(\varphi_{h}, \bar{\psi}_{h} ; \varphi_{h}, \bar{\psi}_{h}\right)=\frac{1}{2} \frac{\mathrm{d}}{\mathrm{d} t} \int_{a}^{b}\left(\varphi_{h}\right)^{2} \mathrm{~d} x+\int_{a}^{b}\left(\bar{\psi}_{h}\right)^{2} \mathrm{~d} x .
$$

We then write the right-hand side of (2.14) as a sum of three terms

$$
B_{j}\left(\varphi^{e}, \psi^{e} ; \varphi_{h}, \bar{\psi}_{h}\right)=B_{j}^{1}+B_{j}^{2}+B_{j}^{3}
$$

where

$$
\begin{gathered}
B_{j}^{1}=\int_{I_{j}} \partial_{t} \varphi^{e} \varphi_{h} \mathrm{~d} x+\int_{I_{j+\frac{1}{2}}} \psi^{e} \bar{\psi}_{h} \mathrm{~d} x \\
B_{j}^{2}=\int_{I_{j}} \psi^{e} \partial_{x} \varphi_{h} \mathrm{~d} x+\int_{I_{j+\frac{1}{2}}} \varphi^{e} \partial_{x} \bar{\psi}_{h} \mathrm{~d} x \\
B_{j}^{3}=-\psi^{e}\left(x_{j+\frac{1}{2}}, t\right) \varphi_{h}\left(x_{j+\frac{1}{2}}^{-}, t\right)+\psi^{e}\left(x_{j-\frac{1}{2}}, t\right) \varphi_{h}\left(x_{j-\frac{1}{2}}^{+}, t\right)-\varphi^{e}\left(x_{j+1}, t\right) \bar{\psi}_{h}\left(x_{j+1}^{-}, t\right)+\varphi^{e}\left(x_{j}, t\right) \bar{\psi}_{h}\left(x_{j}^{+}, t\right)
\end{gathered}
$$

and we will estimate each term separately.

By the definition of the $L^{2}$ projection (2.7)-(2.8), we have

$$
\sum_{j} B_{j}^{1}=0 .
$$

By using the simple inequality

$$
\alpha \beta \leq \frac{1}{2}\left(\alpha^{2}+\beta^{2}\right),
$$

the $L^{2}$ projection error property (2.9)-(2.10) for $\varphi^{e}$ and $\psi^{e}$, and the first inequality in (2.11) for $\varphi_{h}$ and $\bar{\psi}_{h}$, we have:

$$
\sum_{j}\left|B_{j}^{2}\right| \leq \frac{1}{4}\left(\int_{a}^{b}\left(\varphi_{h}\right)^{2} \mathrm{~d} x+\int_{a}^{b}\left(\bar{\psi}_{h}\right)^{2} \mathrm{~d} x\right)+C h^{2 k} .
$$


Finally, by using the simple inequality (2.18), the $L^{2}$ projection error property (2.9)-(2.10) for $\varphi^{e}$ and $\psi^{e}$, and the second inequality in (2.11) for $\varphi_{h}$ and $\bar{\psi}_{h}$, we have:

$$
\sum_{j}\left|B_{j}^{3}\right| \leq \frac{1}{4}\left(\int_{a}^{b}\left(\varphi_{h}\right)^{2} \mathrm{~d} x+\int_{a}^{b}\left(\bar{\psi}_{h}\right)^{2} \mathrm{~d} x\right)+C h^{2 k} .
$$

Summing up (2.17), (2.19) and (2.20) and combining with (2.15), we obtain from (2.14)

$$
\frac{\mathrm{d}}{\mathrm{d} t} \int_{a}^{b}\left(\varphi_{h}\right)^{2} \mathrm{~d} x+\int_{a}^{b}\left(\bar{\psi}_{h}\right)^{2} \mathrm{~d} x \leq C \int_{a}^{b}\left(\varphi_{h}\right)^{2} \mathrm{~d} x+C h^{2 k}
$$

or

Similarly we have:

$$
\frac{\mathrm{d}}{\mathrm{d} t} \int_{a}^{b}\left(P u-u_{h}\right)^{2} \mathrm{~d} x+\int_{a}^{b}\left(Q r-s_{h}\right)^{2} \mathrm{~d} x \leq C \int_{a}^{b}\left(P u-u_{h}\right)^{2} \mathrm{~d} x+C h^{2 k}
$$

$$
\frac{\mathrm{d}}{\mathrm{d} t} \int_{a}^{b}\left(Q u-v_{h}\right)^{2} \mathrm{~d} x+\int_{a}^{b}\left(P r-r_{h}\right)^{2} \mathrm{~d} x \leq C \int_{a}^{b}\left(Q u-v_{h}\right)^{2} \mathrm{~d} x+C h^{2 k} .
$$

This, together with an application of the Gronwall's inequality [1] and the approximation result (2.9), (2.10) for the projection errors and the errors of the initial condition, implies the desired error estimate (2.2).

Remark 2.2. The error estimate of Theorem 2.2 is sub-optimal. The loss of accuracy mainly comes from the estimate on $B_{j}^{2}$. Because it involves the products of piecewise polynomials on different meshes, the standard trick of introducing a projection to make it zero does not apply. The boundary term $B_{j}^{3}$ can be brought to zero by special projections, but this will not help in improving the global error estimate. We will see from numerical experiments later that this suboptimal error estimate is actually sharp for at least $k=1$.

Remark 2.3. The error estimate of Theorem 2.2 can be easily generalized to multi-dimensional scalar linear diffusion equations.

\subsection{A quantitative error estimate via Fourier analysis}

In this subsection we perform a Fourier analysis for the semi-discrete central LDG scheme on overlapping cells (1.7)-(1.10) solving the heat equation (1.2) with uniform meshes for piecewise constant and piecewise linear elements, using the techniques in $[10,11]$, to obtain quantitative error estimates. We also use numerical experiments to verify these error estimates.

For the simplest piecewise constant $k=0$ case, we obtain the finite difference schemes corresponding to the central LDG scheme on overlapping cells (1.7)-(1.10) as follows:

$$
\begin{aligned}
u_{j}^{\prime} & =\frac{1}{h}\left(s_{j+\frac{1}{2}}-s_{j-\frac{1}{2}}\right) \\
s_{j+\frac{1}{2}} & =\frac{1}{h}\left(u_{j+1}-u_{j}\right)
\end{aligned}
$$

for $j=0, \ldots, N-1$. Here $u^{\prime}$ denotes the time derivatives of $u$, and $w_{\alpha}$ denotes the value of the numerical solution $w$ at the point $x_{\alpha}$, with $w=u$ or $s$ and $\alpha=j$ or $j+\frac{1}{2}$. Eliminating $s$, we get:

$$
u_{j}^{\prime}=\frac{1}{h^{2}}\left(u_{j+1}-2 u_{j}+u_{j-1}\right) .
$$

This is apparently the standard second order centered finite difference scheme for solving the heat equation (1.2). Nevertheless, in order to demonstrate our procedure we still perform the following standard Fourier analysis. 
Note that this analysis depends heavily on the assumption of uniform mesh sizes and periodic boundary conditions. We make an ansatz of the form

$$
u_{j}(t)=\hat{u}_{m}(t) \mathrm{e}^{\mathrm{i} m x_{j}}
$$

and substitute this into the scheme (2.24) to find the evolution equation for the coefficient as

$$
\hat{u}_{m}^{\prime}(t)=\frac{2}{h^{2}}(\cos (m h)-1) \hat{u}_{m}(t)
$$

The general solution of the ODE (2.26) is given by

$$
\hat{u}_{m}(t)=a_{0} \mathrm{e}^{\frac{2}{h^{2}}(\cos (m h)-1) t} .
$$

For accuracy we look at the low modes, in particular at $m=1$. To fit the given initial condition

$$
u_{j}(0)=\mathrm{e}^{\mathrm{i} x_{j}}
$$

whose imaginary part is the initial condition

$$
u_{j}(0)=\sin \left(x_{j}\right)
$$

we require, at $t=0$,

$$
\hat{u}_{1}(0)=1
$$

hence we obtain the coefficients $a_{0}$ in $(2.27)$ as

$$
a_{0}=1
$$

We remark that the usual way of taking initial conditions in a finite element method is via an $L^{2}$ projection, not by a point value collocation (2.29), however this does not affect the final results in the analysis in this paper. We thus have the explicit solutions of the scheme (2.24) with the initial condition (2.28), for example

$$
u_{j}(t)=a_{0} \mathrm{e}^{\mathrm{i} x_{j}+\frac{2}{h^{2}}(\cos (m h)-1) t}
$$

with $m=1$ and the coefficient $a_{0}$ given by (2.30). By a simple Taylor expansion, we obtain the imaginary part of $u_{j}(t)$ to be

$$
\operatorname{Im}\left\{u_{j}(t)\right\}=\mathrm{e}^{-t} \sin \left(x_{j}\right)+\frac{1}{12} h^{2} t \mathrm{e}^{-t} \sin \left(x_{j}\right)+O\left(h^{3}\right)
$$

This is clearly consistent, and it is second order accurate at the cell center $x_{j}$. This second order accuracy is however a superconvergence phenomenon for uniform meshes at the cell center. We can do a similar analysis for $\operatorname{Im}\left\{v_{j+\frac{1}{2}}(t)\right\}$.

We now compute the $k=0$ central LDG solutions on overlapping cells $(1.7)-(1.10)$ with $u(x, 0)=\sin (x)$ as the initial condition and with periodic boundary conditions, up to $t=4 \pi$, to verify the quantitative comparison above. We use forward Euler time discretization and take a small time step $\Delta t=0.01 h^{2}$ to reduce the effect from the time discretization. In order to be consistent with the error analysis above, the errors are computed for $u_{h}$ at the points $x_{j}$. The discrete $L^{2}$ and $L^{\infty}$ errors (measuring the errors at cell centers $x_{j}$ only, namely $\sqrt{\frac{1}{N} \sum_{j=1}^{N}\left|u\left(x_{j}, t\right)-u_{j}(t)\right|^{2}}$ for the $L^{2}$ error and $\max _{j}\left|u\left(x_{j}, t\right)-u_{j}(t)\right|$ for the $L^{\infty}$ error) and order of accuracy of the central LDG method on overlapping cells are listed in Table 1. We also list the predicted errors by the analysis, namely the leading terms in the Taylor expansions in (2.32) in the table. We can see that the predicted errors and the actual errors are very close, validating the quantitative analysis in (2.32). The error at cell centers is apparently second order accurate. When measuring as finite element solutions (using 40 uniformly spaced sampling points per cell to compute the $L^{2}$ error $\sqrt{\frac{1}{2 \pi} \int_{0}^{2 \pi}\left|u(x, t)-u_{h}(x, t)\right|^{2} \mathrm{~d} x}$ and the 
TABLE 1. Discrete $L^{2}$ and $L^{\infty}$ errors for $u_{h}$, measured at the center of the cells, and orders of accuracy of the central LDG method (1.7)-(1.10) for $k=0$.

\begin{tabular}{|c|c|c|c|c|c|c|c|c|}
\hline & \multicolumn{4}{|c|}{ Numerical solutions } & \multicolumn{4}{c|}{ Predicted by analysis } \\
\hline$h$ & $L^{2}$ error & Order & $L^{\infty}$ error & Order & $L^{2}$ error & Order & $L^{\infty}$ error & Order \\
\hline $2 \pi / 20$ & $2.68 \mathrm{E}-07$ & - & $3.78 \mathrm{E}-07$ & - & $2.55 \mathrm{E}-07$ & - & $3.60 \mathrm{E}-07$ & - \\
\hline $2 \pi / 40$ & $6.45 \mathrm{E}-08$ & 2.05 & $9.12 \mathrm{E}-08$ & 2.05 & $6.37 \mathrm{E}-08$ & 2.00 & $9.01 \mathrm{E}-01$ & 2.00 \\
\hline $2 \pi / 80$ & $1.60 \mathrm{E}-08$ & 2.01 & $2.26 \mathrm{E}-08$ & 2.01 & $1.59 \mathrm{E}-08$ & 2.00 & $2.25 \mathrm{E}-08$ & 2.00 \\
\hline $2 \pi / 160$ & $3.99 \mathrm{E}-09$ & 2.00 & $5.64 \mathrm{E}-09$ & 2.00 & $3.98 \mathrm{E}-09$ & 2.00 & $5.63 \mathrm{E}-09$ & 2.00 \\
\hline $2 \pi / 320$ & $9.96 \mathrm{E}-10$ & 2.00 & $1.41 \mathrm{E}-09$ & 2.00 & $9.96 \mathrm{E}-10$ & 2.00 & $1.41 \mathrm{E}-09$ & 2.00 \\
\hline $2 \pi / 640$ & $2.49 \mathrm{E}-10$ & 2.00 & $3.52 \mathrm{E}-10$ & 2.00 & $2.49 \mathrm{E}-10$ & 2.00 & $3.52 \mathrm{E}-10$ & 2.00 \\
\hline
\end{tabular}

TABLE 2. $L^{2}$ and $L^{\infty}$ errors, measured as finite element solutions, and orders of accuracy of the central LDG method (1.7)-(1.10) for $k=0$.

\begin{tabular}{|c|c|c|c|c|}
\hline & \multicolumn{5}{|c|}{$u_{h}$} \\
\hline$h$ & $L^{2}$ error & Order & $L^{\infty}$ error & Order \\
\hline $2 \pi / 20$ & $2.01 \mathrm{E}-06$ & - & $6.89 \mathrm{E}-07$ & - \\
\hline $2 \pi / 40$ & $7.08 \mathrm{E}-07$ & 1.51 & $2.92 \mathrm{E}-07$ & 1.24 \\
\hline $2 \pi / 80$ & $3.17 \mathrm{E}-07$ & 1.16 & $1.39 \mathrm{E}-07$ & 1.07 \\
\hline $2 \pi / 160$ & $1.54 \mathrm{E}-07$ & 1.04 & $6.88 \mathrm{E}-08$ & 1.02 \\
\hline $2 \pi / 320$ & $7.67 \mathrm{E}-08$ & 1.01 & $3.43 \mathrm{E}-08$ & 1.00 \\
\hline $2 \pi / 640$ & $3.83 \mathrm{E}-08$ & 1.00 & $1.71 \mathrm{E}-08$ & 1.00 \\
\hline
\end{tabular}

$L^{\infty}$ error $\left.\max _{x}\left|u(x, t)-u_{h}(x, t)\right|\right)$, the $L^{2}$ and $L^{\infty}$ errors and order of accuracy of the central LDG scheme (1.7)-(1.10) are listed in Table 2. Apparently, these errors are first order.

We now repeat this analysis for the piecewise linear $k=1$ case. The solution inside the cell $I_{j}$ or $I_{j+\frac{1}{2}}$ is then represented by

and

$$
u_{h}(x, t)=u_{j-\frac{1}{4}}(t) \varphi_{h}^{1}(\xi)+u_{j+\frac{1}{4}}(t) \varphi_{h}^{2}(\xi)
$$

$$
s_{h}(x, t)=s_{j+\frac{1}{4}}(t) \bar{\psi}_{h}^{1}(\xi)+s_{j+\frac{3}{4}} \bar{\psi}_{h}^{2}(\xi)
$$

where $\varphi_{h}^{1}(\xi)=-\xi+\frac{1}{2}, \varphi_{h}^{2}(\xi)=\xi+\frac{1}{2}, \bar{\psi}_{h}^{1}(\xi)=-\xi+\frac{3}{2}$ and $\bar{\psi}_{h}^{2}(\xi)=\xi-\frac{1}{2}$, with $\xi=\frac{2\left(x-x_{j}\right)}{h}$. With this representation, taking the test functions $\varphi_{h}$ and $\bar{\psi}_{h}$ as $\varphi_{h}^{1}, \varphi_{h}^{2}$ and $\bar{\psi}_{h}^{1}, \bar{\psi}_{h}^{2}$ respectively, eliminating the variable $s$, and inverting the small $2 \times 2$ mass matrix by hand, we obtain easily the finite difference scheme corresponding to the central LDG scheme (1.7) and (1.10) as

$$
\left(\begin{array}{c}
u_{j-\frac{1}{4}}^{\prime} \\
u_{j+\frac{1}{4}}^{\prime}
\end{array}\right)=A\left(\begin{array}{c}
u_{j-\frac{5}{4}} \\
u_{j-\frac{3}{4}}
\end{array}\right)+B\left(\begin{array}{c}
u_{j-\frac{1}{4}} \\
u_{j+\frac{1}{4}}
\end{array}\right)+C\left(\begin{array}{c}
u_{j+\frac{3}{4}} \\
u_{j+\frac{5}{4}}
\end{array}\right) .
$$

with

$$
A=\frac{1}{h^{2}}\left(\begin{array}{cc}
\frac{13}{8} & \frac{-5}{8} \\
\frac{-5}{8} & \frac{13}{8}
\end{array}\right), \quad B=\frac{1}{h^{2}}\left(\begin{array}{cc}
\frac{-13}{4} & \frac{5}{4} \\
\frac{5}{4} & \frac{-13}{4}
\end{array}\right), \quad C=\frac{1}{h^{2}}\left(\begin{array}{cc}
\frac{13}{8} & \frac{-5}{8} \\
\frac{-5}{8} & \frac{13}{8}
\end{array}\right)
$$

for $j=0, \ldots, N-1$. Here $u^{\prime}$ denotes the time derivatives of $u$. We again perform the following standard Fourier analysis for the finite difference scheme (2.33). We make an ansatz of the form

$$
\left(\begin{array}{c}
u_{j-\frac{1}{4}}(t) \\
u_{j+\frac{1}{4}}(t)
\end{array}\right)=\left(\begin{array}{c}
\hat{u}_{m,-\frac{1}{4}}(t) \\
\hat{u}_{m, \frac{1}{4}}(t)
\end{array}\right) \mathrm{e}^{\mathrm{i} m x_{j}}
$$


and substitute this into the scheme (2.33) to find the evolution equation for the coefficient vector as

$$
\left(\begin{array}{c}
\hat{u}_{m,-\frac{1}{4}}^{\prime}(t) \\
\hat{u}_{m,+\frac{1}{4}}^{\prime}(t)
\end{array}\right)=G(m, h)\left(\begin{array}{c}
\hat{u}_{m,-\frac{1}{4}}(t) \\
\hat{u}_{m,+\frac{1}{4}}(t)
\end{array}\right)
$$

where the amplification matrix $G(m, h)$ is given by

$$
G(m, h)=A \mathrm{e}^{-\mathrm{i} m h}+B+C \mathrm{e}^{\mathrm{i} m h}
$$

with the matrices $A, B$ and $C$ defined by (2.34). The eigenvalues of the amplification matrix $G(m, h)$ are

$$
\lambda_{1}=-2+\mathrm{e}^{-\mathrm{i} m h}+\mathrm{e}^{\mathrm{i} m h}, \quad \lambda_{2}=-\frac{9}{4} \mathrm{e}^{-\mathrm{i} m h}\left(-1+\mathrm{e}^{\mathrm{i} m h}\right)^{2} .
$$

The general solution of the ODE (2.33) is given by

$$
\left(\begin{array}{c}
\hat{u}_{m,-\frac{1}{4}}(t) \\
\hat{u}_{m,+\frac{1}{4}}(t)
\end{array}\right)=a_{1} \mathrm{e}^{\lambda_{1} t} V_{1}+a_{2} \mathrm{e}^{\lambda_{2} t} V_{2}
$$

where the eigenvalues $\lambda_{1}$ and $\lambda_{2}$ are given by (2.38), and $V_{1}$ and $V_{2}$ are the corresponding eigenvectors given by

$$
V_{1}=\left(\begin{array}{l}
1 \\
1
\end{array}\right), \quad V_{2}=\left(\begin{array}{c}
-1 \\
1
\end{array}\right) .
$$

For accuracy we look at the low modes, in particular at $m=1$. To fit the given initial condition

$$
u_{j-\frac{1}{4}}(0)=\mathrm{e}^{\mathrm{i} x_{j-\frac{1}{4}}}, \quad u_{j+\frac{1}{4}}(0)=\mathrm{e}^{\mathrm{i} x_{j+\frac{1}{4}}}
$$

whose imaginary part is our initial condition for (1.2), we require, at $t=0$,

$$
\left(\begin{array}{c}
\hat{u}_{m,-\frac{1}{4}}(0) \\
\hat{u}_{m,+\frac{1}{4}}(0)
\end{array}\right)=\left(\begin{array}{c}
\mathrm{e}^{\mathrm{i} \frac{-h}{4}} \\
\mathrm{e}^{\mathrm{i} \frac{h}{4}}
\end{array}\right) .
$$

This gives us the coefficients $a_{1}$ and $a_{2}$ in the solution (2.39). We thus have the explicit solution of the scheme (2.33), (2.34) with the initial condition (2.41), for example

$$
u_{j-\frac{1}{4}}=a_{1} \mathrm{e}^{\mathrm{i} x_{j}+\lambda_{1} t-\mathrm{i} \frac{h}{4}} V_{1}+a_{2} \mathrm{e}^{\mathrm{i} x_{j}+\lambda_{2} t-\mathrm{i} \frac{h}{4}} V_{2}
$$

with the eigenvalues $\lambda_{1}$ and $\lambda_{2}$ given by (2.38) and the eigenvectors $V_{1}$ and $V_{2}$ given by (2.40) with $m=1$, and the coefficients $a_{1}$ and $a_{2}$ obtained by fitting the initial condition. Through a simple Taylor expansion, we obtain the imaginary part of $u_{j-\frac{1}{4}}(t)$ to be

$$
\operatorname{Im}\left\{u_{j-\frac{1}{4}}(t)\right\}=\mathrm{e}^{-t} \sin \left(x_{j-\frac{1}{4}}\right)+\frac{h}{4} \mathrm{e}^{-t}\left(\mathrm{e}^{-\frac{5 t}{4}}+1\right) \cos \left(x_{j-\frac{1}{4}}\right)+O\left(h^{2}\right)
$$

The result for $\operatorname{Im}\left\{u_{j+\frac{1}{4}}(t)\right\}$ is similar. This analysis indicates a sub-optimal convergence rate of first order for piecewise linear polynomials. 
TABLE 3. Discrete $L^{2}$ and $L^{\infty}$ errors for $u_{h}$, measured at the points $x_{j-\frac{1}{4}}$, and orders of accuracy of the central LDG method (1.7)-(1.10) for $k=1$.

\begin{tabular}{|c|c|c|c|c|c|c|c|c|}
\hline & \multicolumn{4}{|c|}{ Numerical solutions } & \multicolumn{4}{c|}{ Predicted by analysis } \\
\hline$h$ & $L^{2}$ error & Order & $L^{\infty}$ error & Order & $L^{2}$ error & Order & $L^{\infty}$ error & Order \\
\hline $2 \pi / 20$ & $3.30 \mathrm{E}-07$ & - & $4.66 \mathrm{E}-07$ & - & $1.94 \mathrm{E}-07$ & - & $2.73 \mathrm{E}-07$ & - \\
\hline $2 \pi / 40$ & $1.16 \mathrm{E}-07$ & 1.50 & $1.64 \mathrm{E}-07$ & 1.50 & $9.68 \mathrm{E}-08$ & 1.00 & $1.37 \mathrm{E}-07$ & 1.00 \\
\hline $2 \pi / 80$ & $5.10 \mathrm{E}-08$ & 1.19 & $7.21 \mathrm{E}-08$ & 1.19 & $4.48 \mathrm{E}-08$ & 1.00 & $6.84 \mathrm{E}-08$ & 1.00 \\
\hline $2 \pi / 160$ & $2.45 \mathrm{E}-08$ & 1.06 & $3.47 \mathrm{E}-08$ & 1.06 & $2.42 \mathrm{E}-08$ & 1.00 & $3.42 \mathrm{E}-08$ & 1.00 \\
\hline $2 \pi / 320$ & $1.22 \mathrm{E}-08$ & 1.01 & $1.72 \mathrm{E}-08$ & 1.01 & $1.21 \mathrm{E}-08$ & 1.00 & $1.71 \mathrm{E}-08$ & 1.00 \\
\hline $2 \pi / 640$ & $6.06 \mathrm{E}-09$ & 1.00 & $8.57 \mathrm{E}-09$ & 1.00 & $6.05 \mathrm{E}-09$ & 1.00 & $8.56 \mathrm{E}-09$ & 1.00 \\
\hline
\end{tabular}

TABLE 4. $L^{2}$ and $L^{\infty}$ errors, measured as finite element solutions, and orders of accuracy of the central LDG method (1.7)-(1.10) for $k=1$.

\begin{tabular}{|c|c|c|c|c|}
\hline & \multicolumn{5}{|c|}{$u_{h}$} \\
\hline$h$ & $L^{2}$ error & Order & $L^{\infty}$ error & Order \\
\hline $2 \pi / 20$ & $1.97 \mathrm{E}-06$ & - & $6.82 \mathrm{E}-07$ & - \\
\hline $2 \pi / 40$ & $7.02 \mathrm{E}-07$ & 1.49 & $2.91 \mathrm{E}-07$ & 1.23 \\
\hline $2 \pi / 80$ & $3.16 \mathrm{E}-07$ & 1.15 & $1.39 \mathrm{E}-07$ & 1.07 \\
\hline $2 \pi / 160$ & $1.54 \mathrm{E}-07$ & 1.04 & $6.87 \mathrm{E}-08$ & 1.02 \\
\hline $2 \pi / 320$ & $7.67 \mathrm{E}-08$ & 1.01 & $3.43 \mathrm{E}-08$ & 1.00 \\
\hline $2 \pi / 640$ & $3.83 \mathrm{E}-08$ & 1.00 & $1.71 \mathrm{E}-08$ & 1.00 \\
\hline
\end{tabular}

In principle this analysis can be performed for higher order polynomials in the central LDG scheme on overlapping cells (1.7)-(1.10), however the algebra becomes prohibitively complicated.

We now compute the central LDG scheme (1.7)-(1.10) for $k=1$ on overlapping cells to $(1.2)$ with $u(x, 0)=$ $\sin (x)$ as the initial condition and with periodic boundary conditions, up to $t=4 \pi$, to verify the quantitative analysis above. We use a second order Runge-Kutta method and take a small time step $\Delta t=0.01 h^{2}$ to reduce the effect from the time discretization. In order to be consistent with the error analysis above, the errors are computed for $u_{h}$ at the points $x_{j-\frac{1}{4}}$. The discrete $L^{2}$ and $L^{\infty}$ errors and order of accuracy of the central LDG method (1.7)-(1.10) on overlapping cells are listed in Table 3. We also list the predicted errors by the analysis, namely the leading terms in the Taylor expansions in (2.43) in the tables. We can see that the predicted errors and the actual errors are very close, validating our quantitative analysis in (2.43). It is only first order accurate for the LDG method on overlapping cells. When measuring as finite element solutions (using 40 uniformly spaced sampling points per cell), the $L^{2}$ and $L^{\infty}$ errors and order of accuracy of the central LDG method (1.7)-(1.10) are listed in Table 4. Again, the errors are only first order.

According to the analysis and numerical experiments in this section and in Section 4, it appears that the central LDG method (1.7)-(1.10) on overlapping cells is suboptimal of order $k$ for $k=1$ but it seems to achieve the optimal $(k+1)$-th order for $k>1$.

\section{Analysis of the SECOND Version of CENTRAL LDG SCHEME ON OVERLAPPing CELLS}

In this section, we perform a similar analysis for the second version of the central LDG scheme on overlapping cells, given by (1.11)-(1.14), for solving the heat equation (1.2). As before, we study the $L^{2}$ stability of the central LDG scheme (1.11)-(1.14) in Section 3.1. In Section 3.2 we provide a $L^{2}$ a priori error estimate for 
smooth solutions. In Section 3.3 we give a quantitative error estimate for this scheme for polynomial degree up to 1 using Fourier analysis, and provide numerical evidence to verify this analysis.

\section{1. $L^{2}$ stability}

Theorem 3.1. The numerical solution $u_{h}, r_{h}, v_{h}$ and $s_{h}$ of the central LDG scheme (1.11)-(1.14) for equation (1.2) satisfies the following $L^{2}$ stability condition

$$
\frac{1}{2} \frac{\mathrm{d}}{\mathrm{d} t} \int_{a}^{b}\left(\left(u_{h}\right)^{2}+\left(v_{h}\right)^{2}\right) \mathrm{d} x+\int_{a}^{b}\left(r_{h}^{2}+s_{h}^{2}\right) \mathrm{d} x \leq 0
$$

Proof. Taking the test functions $\varphi_{h}=u_{h}, \psi_{h}=v_{h}, \bar{\varphi}_{h}=r_{h}$ and $\bar{\psi}_{h}=s_{h}$ in (1.11)-(1.14) respectively, summing up over $j$, and observing the periodic boundary condition, we have

$$
\begin{aligned}
\frac{1}{2} & \frac{\mathrm{d}}{\mathrm{d} t} \int_{a}^{b} u_{h}^{2} \mathrm{~d} x+\frac{1}{2} \frac{\mathrm{d}}{\mathrm{d} t} \int_{a}^{b} v_{h}^{2} \mathrm{~d} x+\int_{a}^{b} r_{h}^{2} \mathrm{~d} x+\int_{a}^{b} s_{h}^{2} \mathrm{~d} x \\
= & \sum_{j}\left[\frac{1}{\tau_{\max }}\left(\int_{x_{j-\frac{1}{2}}}^{x_{j+\frac{1}{2}}}\left(v_{h}-u_{h}\right) u_{h} \mathrm{~d} x+\int_{x_{j}}^{x_{j+1}}\left(u_{h}-v_{h}\right) v_{h} \mathrm{~d} x\right)\right. \\
& -\left(\int_{x_{j-\frac{1}{2}}}^{x_{j+\frac{1}{2}}} \partial_{x}\left(u_{h}\right) s_{h} \mathrm{~d} x+\int_{x_{j}}^{x_{j+1}} \partial_{x}\left(s_{h}\right) u_{h} \mathrm{~d} x\right)-\left(\int_{x_{j-\frac{1}{2}}}^{x_{j+\frac{1}{2}}} \partial_{x}\left(r_{h}\right) v_{h} \mathrm{~d} x+\int_{x_{j}}^{x_{j+1}} \partial_{x}\left(v_{h}\right) r_{h} \mathrm{~d} x\right) \\
& +s_{h}\left(x_{j+\frac{1}{2}}, t\right) u_{h}\left(x_{j+\frac{1}{2}}^{-}, t\right)-s_{h}\left(x_{j-\frac{1}{2}}, t\right) u_{h}\left(x_{j-\frac{1}{2}}^{+}, t\right)+r_{h}\left(x_{j+1}, t\right) v_{h}\left(x_{j+1}^{-}, t\right) \\
& -r_{h}\left(x_{j}, t\right) v_{h}\left(x_{j}^{+}, t\right)+v_{h}\left(x_{j+\frac{1}{2}}, t\right) r_{h}\left(x_{j+\frac{1}{2}}^{-}, t\right)-v_{h}\left(x_{j-\frac{1}{2}}, t\right) r_{h}\left(x_{j-\frac{1}{2}}^{+}, t\right) \\
& \left.+u_{h}\left(x_{j+1}, t\right) s_{h}\left(x_{j+1}^{-}, t\right)-u_{h}\left(x_{j}, t\right) s_{h}\left(x_{j}^{+}, t\right)\right] \\
= & -\frac{1}{\tau_{\max }} \int_{a}^{b}\left(u_{h}-v_{h}\right)^{2} \mathrm{~d} x \\
\leq & 0 .
\end{aligned}
$$

Remark 3.1. Comparing with the $L^{2}$ stability of the first version of the central LDG scheme, we notice the additional numerical dissipation $\int_{a}^{b}\left(u_{h}-v_{h}\right)^{2} \mathrm{~d} x$, which is related to the difference of the two duplicative sets of information in the computational domain. The second version of the central LDG scheme couples these two sets of duplicative solutions through the extra numerical diffusion term.

\section{2. $L^{2}$ a priori error estimate}

In this subsection we obtain an a priori $L^{2}$ error estimate for the central LDG scheme (1.11)-(1.14).

Theorem 3.2. The numerical solution $u_{h}, r_{h}, v_{h}$ and $s_{h}$ of the central LDG scheme (1.11)-(1.14) for the equation (1.2) with a smooth initial condition $u(\cdot, 0) \in H^{k+2}$ satisfies the following $L^{2}$ error estimate

$$
\left\|u-u_{h}\right\|^{2}+\left\|u-v_{h}\right\|^{2}+\left\|\left|u_{x}-r_{h}\right|\right\|^{2}+\left\|\left|u_{x}-s_{h}\right|\right\|^{2} \leq C h^{2 k}
$$

where $u$, the constant $C$, and the norms are the same as those in Theorem 2.2. 
Proof. We introduce the following notation

$$
\begin{aligned}
& B_{j}\left(u_{h}, v_{h}, r_{h}, s_{h} ; \varphi_{h}, \psi_{h}, \bar{\varphi}_{h}, \bar{\psi}_{h}\right) \\
& =\int_{I_{j}} \partial_{t}\left(u_{h}\right) \varphi_{h} \mathrm{~d} x+\int_{I_{j+\frac{1}{2}}} \partial_{t}\left(v_{h}\right) \psi_{h} \mathrm{~d} x+\int_{I_{j}} r_{h} \bar{\varphi}_{h} \mathrm{~d} x+\int_{I_{j+\frac{1}{2}}} s_{h} \bar{\psi}_{h} \mathrm{~d} x \\
& \quad-\frac{1}{\tau_{\max }}\left(\int_{I_{j}}\left(v_{h}-u_{h}\right) \varphi_{h} \mathrm{~d} x+\int_{j+\frac{1}{2}}\left(u_{h}-v_{h}\right) \psi_{h} \mathrm{~d} x\right) \\
& \quad+\int_{I_{j}} \partial_{x}\left(\varphi_{h}\right) s_{h} \mathrm{~d} x+\int_{I_{j+\frac{1}{2}}} \partial_{x}\left(\psi_{h}\right) r_{h} \mathrm{~d} x+\int_{I_{j}} \partial_{x}\left(\bar{\varphi}_{h}\right) v_{h} \mathrm{~d} x+\int_{I_{j+\frac{1}{2}}} \partial_{x}\left(\bar{\psi}_{h}\right) u_{h} \mathrm{~d} x \\
& \quad-s_{h}\left(x_{j+\frac{1}{2}}, t\right) \varphi_{h}\left(x_{j+\frac{1}{2}}^{-}, t\right)+s_{h}\left(x_{j-\frac{1}{2}}, t\right) \varphi_{h}\left(x_{j-\frac{1}{2}}^{+}, t\right)-r_{h}\left(x_{j+1}, t\right) \psi_{h}\left(x_{j+1}^{-}, t\right) \\
& \quad+r_{h}\left(x_{j}, t\right) \psi_{h}\left(x_{j}^{+}, t\right)-v_{h}\left(x_{j+\frac{1}{2}}, t\right) \bar{\varphi}_{h}\left(x_{j+\frac{1}{2}}^{-}, t\right)+v_{h}\left(x_{j-\frac{1}{2}}, t\right) \bar{\varphi}_{h}\left(x_{j-\frac{1}{2}}^{+}, t\right) \\
& \quad-u_{h}\left(x_{j+1}, t\right) \bar{\psi}_{h}\left(x_{j+1}^{-}, t\right)+u_{h}\left(x_{j}, t\right) \bar{\psi}_{h}\left(x_{j}^{+}, t\right) .
\end{aligned}
$$

By the definition of the scheme, we have:

$$
B_{j}\left(u_{h}, v_{h}, r_{h}, s_{h} ; \varphi_{h}, \psi_{h}, \bar{\varphi}_{h}, \bar{\psi}_{h}\right)=0
$$

for all $j$ and all $\varphi_{h}, \bar{\varphi}_{h} \in V_{h}$ and $\psi_{h}, \bar{\psi}_{h} \in W_{h}$. The consistency of the scheme can also be easily verified, namely the exact solutions $u, v=u$ and $r, s=u_{x}$ of the PDE (1.2) satisfy

$$
B_{j}\left(u, v, r, s ; \varphi_{h}, \psi_{h}, \bar{\varphi}_{h}, \bar{\psi}_{h}\right)=0
$$

for all $j$ and all $\varphi_{h}, \bar{\varphi}_{h} \in V_{h}$ and $\psi_{h}, \bar{\psi}_{h} \in W_{h}$. Subtracting (3.3) from (3.4), we obtain the error equation or the Galerkin orthogonality

$$
B_{j}\left(u-u_{h}, v-v_{h}, r-r_{h}, s-s_{h} ; \varphi_{h}, \psi_{h}, \bar{\varphi}_{h}, \bar{\psi}_{h}\right)=0
$$

for all $\varphi_{h}, \bar{\varphi}_{h} \in V_{h}$ and $\psi_{h}, \bar{\psi}_{h} \in W_{h}$.

With $P$ and $Q$ still denoting the standard $L^{2}$ projection into $V_{h}$ and $W_{h}$ respectively, we take

$$
\varphi_{h}=P u-u_{h}, \quad \psi_{h}=Q v-v_{h}, \quad \bar{\varphi}_{h}=P r-r_{h}, \quad \bar{\psi}_{h}=Q s-s_{h}
$$

in the error equation (3.5), and denote

$$
\varphi^{e}=P u-u, \quad \psi^{e}=Q v-v, \quad \bar{\varphi}^{e}=P r-r, \quad \bar{\psi}^{e}=Q s-s
$$

to obtain

$$
B_{j}\left(\varphi_{h}, \psi_{h}, \bar{\varphi}_{h}, \bar{\psi}_{h} ; \varphi_{h}, \psi_{h}, \bar{\varphi}_{h}, \bar{\psi}_{h}\right)=B_{j}\left(\varphi^{e}, \psi^{e}, \bar{\varphi}^{e}, \bar{\psi}^{e} ; \varphi_{h}, \psi_{h}, \bar{\varphi}_{h}, \bar{\psi}_{h}\right) .
$$

For the left-hand side of (3.8), we use a similar proof as that for Theorem 3.1 to conclude

$$
\begin{aligned}
& \sum_{j} B_{j}\left(\varphi_{h}, \psi_{h}, \bar{\varphi}_{h}, \bar{\psi}_{h} ; \varphi_{h}, \psi_{h}, \bar{\varphi}_{h}, \bar{\psi}_{h}\right) \\
= & \frac{1}{2} \frac{\mathrm{d}}{\mathrm{d} t} \int_{a}^{b}\left(\varphi_{h}\right)^{2} \mathrm{~d} x+\frac{1}{2} \frac{\mathrm{d}}{\mathrm{d} t} \int_{a}^{b}\left(\psi_{h}\right)^{2} \mathrm{~d} x+\int_{a}^{b}\left(\bar{\varphi}_{h}\right)^{2} \mathrm{~d} x+\int_{a}^{b}\left(\bar{\psi}_{h}\right)^{2} \mathrm{~d} x \\
& +\frac{1}{\tau_{\max }} \int_{a}^{b}\left(\varphi_{h}-\psi_{h}\right)^{2} \mathrm{~d} x .
\end{aligned}
$$


We write the right-hand side of (3.8) as a sum of four terms

$$
B_{j}\left(\varphi^{e}, \psi^{e}, \bar{\varphi}^{e}, \bar{\psi}^{e} ; \varphi_{h}, \psi_{h}, \bar{\varphi}_{h}, \bar{\psi}_{h}\right)=B_{j}^{1}+B_{j}^{2}+B_{j}^{3}+B_{j}^{4}
$$

where

$$
\begin{aligned}
B_{j}^{1}= & \int_{I_{j}} \partial_{t} \varphi^{e} \varphi_{h} \mathrm{~d} x+\int_{I_{j+\frac{1}{2}}} \partial_{t} \psi^{e} \psi_{h} \mathrm{~d} x+\int_{I_{j}} \bar{\varphi}^{e} \bar{\varphi}_{h} \mathrm{~d} x+\int_{I_{j+\frac{1}{2}}} \bar{\psi}^{e} \bar{\psi}_{h} \mathrm{~d} x \\
B_{j}^{2}= & -\frac{1}{\tau_{\max }}\left(\int_{I_{j}}\left(\psi^{e}-\varphi^{e}\right) \varphi_{h} \mathrm{~d} x+\int_{j+\frac{1}{2}}\left(\varphi^{e}-\psi^{e}\right) \psi_{h} \mathrm{~d} x\right) \\
B_{j}^{3}= & \int_{I_{j}} \partial_{x}\left(\varphi_{h}\right) \bar{\psi}^{e} \mathrm{~d} x+\int_{I_{j+\frac{1}{2}}} \partial_{x}\left(\psi_{h}\right) \bar{\varphi}^{e} \mathrm{~d} x+\int_{I_{j}} \partial_{x}\left(\bar{\varphi}_{h}\right) \psi^{e} \mathrm{~d} x+\int_{I_{j+\frac{1}{2}}} \partial_{x}\left(\bar{\psi}_{h}\right) \varphi^{e} \mathrm{~d} x \\
B_{j}^{4}= & -\bar{\psi}^{e}\left(x_{j+\frac{1}{2}}, t\right) \varphi_{h}\left(x_{j+\frac{1}{2}}^{-}, t\right)+\bar{\psi}^{e}\left(x_{j-\frac{1}{2}}, t\right) \varphi_{h}\left(x_{j-\frac{1}{2}}^{+}, t\right)-\bar{\varphi}^{e}\left(x_{j+1}, t\right) \psi_{h}\left(x_{j+1}^{-}, t\right) \\
& +\bar{\varphi}^{e}\left(x_{j}, t\right) \psi_{h}\left(x_{j}^{+}, t\right)-\psi^{e}\left(x_{j+\frac{1}{2}}, t\right) \bar{\varphi}_{h}\left(x_{j+\frac{1}{2}}^{-}, t\right)+\psi^{e}\left(x_{j-\frac{1}{2}}, t\right) \bar{\varphi}_{h}\left(x_{j-\frac{1}{2}}^{+}, t\right) \\
& -\varphi^{e}\left(x_{j+1}, t\right) \bar{\psi}_{h}\left(x_{j+1}^{-}, t\right)+\varphi^{e}\left(x_{j}, t\right) \bar{\psi}_{h}\left(x_{j}^{+}, t\right)
\end{aligned}
$$

and we will estimate each term separately.

By the definition of the $L^{2}$ projection (2.7), (2.8), we have

$$
\sum_{j} B_{j}^{1}=0 .
$$

By using the simple inequality (2.18), the $L^{2}$ projection error property (2.9), (2.10) for $\varphi^{e}$ and $\psi^{e}$, and the fact that $\tau_{\max }=c h^{2}$, we have

$$
\begin{aligned}
\sum_{j}\left|B_{j}^{2}\right| & \leq \frac{1}{\tau_{\max }}\left(\frac{1}{2} \int_{a}^{b}\left(\psi^{e}-\varphi^{e}\right)^{2} \mathrm{~d} x+\frac{1}{2} \int_{a}^{b}\left(\varphi_{h}-\psi_{h}\right)^{2} \mathrm{~d} x\right) \\
& \leq \frac{1}{2 \tau_{\max }} \int_{a}^{b}\left(\varphi_{h}-\psi_{h}\right)^{2} \mathrm{~d} x+C h^{2 k} .
\end{aligned}
$$

Again, by using the simple inequality (2.18), the $L^{2}$ projection error property $(2.9),(2.10)$ for $\varphi^{e}, \psi^{e}, \bar{\varphi}^{e}$ and $\bar{\psi}^{e}$, and the first inequality in (2.11) for $\varphi_{h}, \psi_{h}, \bar{\varphi}_{h}$ and $\bar{\psi}_{h}$, we have:

$$
\sum_{j}\left|B_{j}^{3}\right| \leq \frac{1}{4} \int_{a}^{b}\left(\left(\varphi_{h}\right)^{2} \mathrm{~d} x+\left(\psi_{h}\right)^{2}+\left(\bar{\varphi}_{h}\right)^{2}+\left(\bar{\psi}_{h}\right)^{2}\right) \mathrm{d} x+C h^{2 k} .
$$

Finally, by using the simple inequality (2.18), the $L^{2}$ projection error property (2.9)-(2.10) for $\varphi^{e}, \psi^{e}, \bar{\varphi}^{e}$ and $\bar{\psi}^{e}$, and the second inequality in (2.11) for $\varphi_{h}, \psi_{h}, \bar{\varphi}_{h}$ and $\bar{\psi}_{h}$, we have:

$$
\sum_{j}\left|B_{j}^{4}\right| \leq \frac{1}{4} \int_{a}^{b}\left(\left(\varphi_{h}\right)^{2} \mathrm{~d} x+\left(\psi_{h}\right)^{2}+\left(\bar{\varphi}_{h}\right)^{2}+\left(\bar{\psi}_{h}\right)^{2}\right) \mathrm{d} x+C h^{2 k}
$$

Summing up (3.11), (3.12), (3.13) and (3.14) and combining with (3.9), we obtain from (3.8)

$$
\frac{\mathrm{d}}{\mathrm{d} t} \int_{a}^{b}\left(\left(\varphi_{h}\right)^{2}+\left(\psi_{h}\right)^{2}\right) \mathrm{d} x+\int_{a}^{b}\left(\left(\bar{\varphi}_{h}\right)^{2}+\left(\bar{\psi}_{h}\right)^{2}\right) \mathrm{d} x \leq \int_{a}^{b}\left(\left(\varphi_{h}\right)^{2}+\left(\psi_{h}\right)^{2}\right) \mathrm{d} x+C h^{2 k} .
$$


This, together with an application of the Gronwall's inequality and the approximation result (2.9), (2.10) for the projection errors and the errors of the initial condition, implies the desired error estimate (3.2).

Remark 3.2. The error estimate of Theorem 3.2 is sub-optimal. Analysis in the next subsection and numerical evidence there and in Section 4 indicate that the method actually converges at the optimal $(k+1)$-th order.

Remark 3.3. The error estimate of Theorem 3.2 can be easily generalized to multi-dimensional scalar linear diffusion equations.

\subsection{A quantitative error estimate via Fourier analysis}

In this subsection we perform a Fourier analysis for the second version of the semi-discrete central LDG scheme (1.11)-(1.14) for the diffusion equation (1.2) with uniform meshes for piecewise constant and linear elements, using the techniques in $[10,11]$ like in Section 2.3. We also use numerical experiments to verify these error estimates.

For the piecewise constant $k=0$ case, we obtain the finite difference schemes corresponding to the central LDG scheme (1.11)-(1.14) as follows:

$$
\begin{aligned}
u_{j}^{\prime} & =\frac{1}{h}\left(s_{j+\frac{1}{2}}-s_{j-\frac{1}{2}}\right)-\frac{1}{\tau_{\max }} u_{j}+\frac{1}{2 \tau_{\max }}\left(v_{j+\frac{1}{2}}-v_{j-\frac{1}{2}}\right) \\
r_{j} & =\frac{1}{h}\left(v_{j+\frac{1}{2}}-v_{j-\frac{1}{2}}\right) \\
v_{j+\frac{1}{2}}^{\prime} & =\frac{1}{h}\left(r_{j+1}-r_{j}\right)-\frac{1}{\tau_{\max }} v_{j+\frac{1}{2}}+\frac{1}{2 \tau_{\max }}\left(u_{j+1}-u_{j}\right) \\
s_{j+\frac{1}{2}} & =\frac{1}{h}\left(u_{j+1}-u_{j}\right)
\end{aligned}
$$

for $j=0, \ldots, N-1$. Here $u^{\prime}$ and $v^{\prime}$ denotes the time derivatives of $u$ and $v$ respectively. Eliminating $r$ and $s$, we get:

$$
\left(\begin{array}{c}
u_{j}^{\prime} \\
v_{j+\frac{1}{2}}^{\prime}
\end{array}\right)=A\left(\begin{array}{c}
u_{j-1} \\
v_{j-\frac{1}{2}}
\end{array}\right)+B\left(\begin{array}{c}
u_{j} \\
v_{j+\frac{1}{2}}
\end{array}\right)+C\left(\begin{array}{c}
u_{j+1} \\
v_{j+\frac{3}{2}}
\end{array}\right)
$$

with

$$
A=\frac{1}{h^{2}}\left(\begin{array}{cc}
1 & \frac{h^{2}}{2 \tau_{\max }} \\
0 & 1
\end{array}\right), \quad B=\frac{1}{h^{2}}\left(\begin{array}{cc}
-2-\frac{h^{2}}{\tau_{\max }} & \frac{h^{2}}{2 \tau_{\max }} \\
\frac{h^{2}}{2 \tau_{\max }} & -2-\frac{h^{2}}{\tau_{\max }}
\end{array}\right), \quad C=\frac{1}{h^{2}}\left(\begin{array}{cc}
1 & 0 \\
\frac{h^{2}}{2 \tau_{\max }} & 1
\end{array}\right)
$$

for $j=0, \ldots, N-1$. We again perform the following standard Fourier analysis for the finite difference scheme (3.16). We make an ansatz of the form

$$
\left(\begin{array}{c}
u_{j}(t) \\
v_{j+\frac{1}{2}}(t)
\end{array}\right)=\left(\begin{array}{c}
\hat{u}_{m, 0}(t) \\
\hat{v}_{m, \frac{1}{2}}(t)
\end{array}\right) \mathrm{e}^{\mathrm{i} m x_{j}}
$$

and substitute this into the scheme (3.16) to find the evolution equation for the coefficient vector as

$$
\left(\begin{array}{c}
\hat{u}_{m, 0}^{\prime}(t) \\
\hat{v}_{m, \frac{1}{2}}^{\prime}(t)
\end{array}\right)=G(m, h)\left(\begin{array}{c}
\hat{u}_{m, 0}(t) \\
\hat{u}_{m, \frac{1}{2}}(t)
\end{array}\right)
$$

where the amplification matrix $G(m, h)$ is given by

$$
G(m, h)=A \mathrm{e}^{-\mathrm{i} m h}+B+C \mathrm{e}^{\mathrm{i} m h}
$$


TABLE 5. Discrete $L^{2}$ and $L^{\infty}$ errors for $u_{h}$, measured at the center of the cells, and orders of accuracy of the central LDG method (1.11)-(1.14) for $k=0$.

\begin{tabular}{|c|c|c|c|c|c|c|c|c|}
\hline & \multicolumn{4}{|c|}{ Numerical solutions } & \multicolumn{4}{c|}{ Predicted by analysis } \\
\hline$h$ & $L^{2}$ error & Order & $L^{\infty}$ error & Order & $L^{2}$ error & Order & $L^{\infty}$ error & Order \\
\hline $2 \pi / 20$ & $2.47 \mathrm{E}-06$ & - & $3.49 \mathrm{E}-06$ & - & $2.47 \mathrm{E}-06$ & - & $3.49 \mathrm{E}-06$ & - \\
\hline $2 \pi / 40$ & $2.47 \mathrm{E}-06$ & 0.00 & $3.49 \mathrm{E}-06$ & 0.00 & $2.47 \mathrm{E}-06$ & 0.00 & $3.49 \mathrm{E}-06$ & 0.00 \\
\hline $2 \pi / 80$ & $2.47 \mathrm{E}-06$ & 0.00 & $3.49 \mathrm{E}-06$ & 0.00 & $2.47 \mathrm{E}-06$ & 0.00 & $3.49 \mathrm{E}-06$ & 0.00 \\
\hline $2 \pi / 160$ & $2.47 \mathrm{E}-06$ & 0.00 & $3.49 \mathrm{E}-06$ & 0.00 & $2.47 \mathrm{E}-06$ & 0.00 & $3.49 \mathrm{E}-06$ & 0.00 \\
\hline $2 \pi / 320$ & $2.47 \mathrm{E}-06$ & 0.00 & $3.49 \mathrm{E}-06$ & 0.00 & $2.47 \mathrm{E}-06$ & 0.00 & $3.49 \mathrm{E}-06$ & 0.00 \\
\hline $2 \pi / 640$ & $2.47 \mathrm{E}-06$ & 0.00 & $3.49 \mathrm{E}-06$ & 0.00 & $2.47 \mathrm{E}-06$ & 0.00 & $3.49 \mathrm{E}-06$ & 0.00 \\
\hline
\end{tabular}

with the matrices $A, B$ and $C$ defined by (3.17). For accuracy we look at the low modes, in particular at $m=1$. To fit the given initial condition

$$
u_{j}(0)=\mathrm{e}^{\mathrm{i} x_{j}}, \quad v_{j+\frac{1}{2}}(0)=\mathrm{e}^{\mathrm{i} x_{j+\frac{1}{2}}}
$$

whose imaginary part is our initial condition for (1.2), we require, at $t=0$,

$$
\left(\begin{array}{c}
\hat{u}_{m, 0}(0) \\
\hat{v}_{m, \frac{1}{2}}(0)
\end{array}\right)=\left(\begin{array}{c}
1 \\
\mathrm{e}^{\mathrm{i} \frac{h}{2}}
\end{array}\right)
$$

Then we get the solution of the ODEs (3.16), (3.17) with the initial condition (3.21) for $m=1$. Through a simple Taylor expansion, we obtain the imaginary part of $u_{j}(t)$ to be

$$
\operatorname{Im}\left\{u_{j}(t)\right\}=\mathrm{e}^{-t-\frac{t}{8 a}} \sin \left(x_{j}\right)+h^{2}\left(\frac{1}{12} \mathrm{e}^{-t-\frac{t}{8 a}}+\frac{t}{384 a} \mathrm{e}^{-t-\frac{t}{8 a}}\right) \sin \left(x_{j}\right)+O\left(h^{3}\right)
$$

where $a=\frac{\tau_{\max }}{h^{2}}$. The result for $\operatorname{Im}\left\{v_{j+\frac{1}{2}}(t)\right\}$ is similar. This analysis indicates that the scheme is not consistent for piecewise constant elements.

We now compute the central LDG solutions on overlapping cells $(1.11)-(1.14)$ with $u(x, 0)=\sin (x)$ as the initial condition and with periodic boundary conditions, up to $t=4 \pi$, to verify the quantitative analysis above. Numerical results show that the maximum time step for stability is $\tau_{\max }=0.12 h^{2}$. We use forward Euler time discretization and take a small time step $\Delta t=0.1 \tau_{\max }$ to reduce the effect from the time discretization. In order to be consistent with the error analysis above, the errors are computed for $u_{h}$ at the points $x_{j}$ for the piecewise constant elements. The numerical results are listed in Table 5. We also list the predicted errors by the analysis, namely the leading terms in the Taylor expansions in (3.22) in the table. We can see that the error analysis above and the numerical results are consistent, indicating that the method for the $k=0$ case is not consistent.

For the piecewise linear $k=1$ case, we obtain easily the finite difference scheme corresponding to the central LDG scheme (1.11)-(1.14) overlapping cells:

$$
\left(\begin{array}{c}
u_{j-\frac{1}{4}}^{\prime} \\
u_{j+\frac{1}{4}}^{\prime} \\
v_{j+\frac{1}{4}}^{\prime} \\
v_{j+\frac{3}{4}}^{\prime}
\end{array}\right)=A\left(\begin{array}{c}
u_{j-\frac{5}{4}} \\
u_{j-\frac{3}{4}} \\
v_{j-\frac{3}{4}} \\
v_{j-\frac{1}{4}}
\end{array}\right)+B\left(\begin{array}{c}
u_{j-\frac{1}{4}} \\
u_{j+\frac{1}{4}} \\
v_{j+\frac{1}{4}} \\
v_{j+\frac{3}{4}}
\end{array}\right)+C\left(\begin{array}{c}
u_{j+\frac{3}{4}} \\
u_{j+\frac{5}{4}} \\
v_{j+\frac{5}{4}} \\
v_{j+\frac{7}{4}}
\end{array}\right)
$$


TABLE 6. Discrete $L^{2}$ and $L^{\infty}$ errors and orders of accuracy of the central LDG method (1.11)(1.14), measured at the points $x_{j-\frac{1}{4}}$ for $k=1$.

\begin{tabular}{|c|c|c|c|c|c|c|c|c|}
\hline & \multicolumn{4}{|c|}{ Numerical solutions } & \multicolumn{4}{c|}{ Predicted by analysis } \\
\hline$h$ & $L^{2}$ error & Order & $L^{\infty}$ error & Order & $L^{2}$ error & Order & $L^{\infty}$ error & Order \\
\hline $2 \pi / 20$ & $7.18 \mathrm{E}-07$ & - & $1.01 \mathrm{E}-06$ & - & $8.60 \mathrm{E}-07$ & - & $1.21 \mathrm{E}-06$ & - \\
\hline $2 \pi / 40$ & $2.05 \mathrm{E}-07$ & 1.81 & $2.90 \mathrm{E}-07$ & 1.80 & $2.15 \mathrm{E}-07$ & 2.00 & $3.04 \mathrm{E}-07$ & 2.00 \\
\hline $2 \pi / 80$ & $5.31 \mathrm{E}-08$ & 1.95 & $7.51-08$ & 1.95 & $5.38 \mathrm{E}-08$ & 2.00 & $7.60 \mathrm{E}-08$ & 2.00 \\
\hline $2 \pi / 160$ & $1.34 \mathrm{E}-08$ & 1.99 & $1.90 \mathrm{E}-08$ & 1.99 & $1.34 \mathrm{E}-08$ & 2.00 & $1.90 \mathrm{E}-08$ & 2.00 \\
\hline $2 \pi / 320$ & $3.36 \mathrm{E}-09$ & 2.00 & $4.75 \mathrm{E}-09$ & 2.00 & $3.36 \mathrm{E}-09$ & 2.00 & $4.75 \mathrm{E}-09$ & 2.00 \\
\hline $2 \pi / 640$ & $8.40 \mathrm{E}-10$ & 2.00 & $1.19 \mathrm{E}-09$ & 2.00 & $8.40 \mathrm{E}-10$ & 2.00 & $1.19 \mathrm{E}-09$ & 2.00 \\
\hline
\end{tabular}

with the matrices $A, B$, and $C$ given as

$$
\begin{aligned}
& A=\frac{1}{16 a}\left(\begin{array}{cccc}
26 a & -10 a & 1 & 13 \\
-10 a & 26 a & -1 & 3 \\
0 & 0 & 26 a & -10 a \\
0 & 0 & -10 a & 26 a
\end{array}\right) \\
& B=\frac{1}{16 a}\left(\begin{array}{cccc}
-4(4+13 a) & 20 a & 3 & -1 \\
20 a & -4(4+13 a) & 13 & 1 \\
1 & 13 & -4(4+13 a) & 20 a \\
-1 & 3 & 20 a & -4(4+13 a)
\end{array}\right) \\
& C=\frac{1}{16 a}\left(\begin{array}{cccc}
26 a & -10 a & 0 & 0 \\
-10 a & 26 a & 0 & 0 \\
3 & -1 & 26 a & -10 a \\
13 & 1 & -10 a & 26 a
\end{array}\right)
\end{aligned}
$$

for $j=0, \ldots, N-1$, where $a=\frac{\tau_{\max }}{h^{2}}$. Here $u^{\prime}$ and $v^{\prime}$ denote the time derivatives of $u$ and $v$ respectively. We again perform the standard Fourier analysis for the finite difference scheme. We obtain the imaginary part of $u_{j-\frac{1}{4}}(t)$ to be

$$
\operatorname{Im}\left\{u_{j-\frac{1}{4}}(t)\right\}=\mathrm{e}^{-t} \sin \left(x_{j-\frac{1}{4}}\right)+\frac{(1+8 a) t}{384 a} h^{2} \mathrm{e}^{-t} \sin \left(x_{j-\frac{1}{4}}\right)+O\left(h^{3}\right) .
$$

The result for $\operatorname{Im}\left\{u_{j+\frac{1}{4}}(t)\right\}, \operatorname{Im}\left\{v_{j+\frac{1}{4}}(t)\right\}$ and $\operatorname{Im}\left\{v_{j+\frac{3}{4}}(t)\right\}$ are similar. Clearly, the method is now optimal second order accurate.

We now compute the central LDG scheme (1.11)-(1.14) on overlapping cells to (1.2) with $u(x, 0)=\sin (x)$ as the initial condition and with periodic boundary conditions, up to $t=4 \pi$, to verify the quantitative analysis above. We take $\tau_{\max }=0.01 h^{2}$, and use a second order Runge-Kutta method and take a small time step $\Delta t=0.1 \tau_{\max }$ to reduce the effect from the time discretization. In order to be consistent with the error analysis above, the errors are computed for $u_{h}$ at the points $x_{j-\frac{1}{4}}$. The discrete $L^{2}$ and $L^{\infty}$ errors and order of accuracy of the central LDG method (1.11)-(1.14) are listed in Table 6 . We also list the predicted errors by the analysis, namely the leading terms in the Taylor expansions in (3.25) in the table. We can see that the predicted errors and the actual errors are very close, validating our quantitative analysis in (3.25). It clear shows the expected optimal second order accuracy. When measuring as finite element solutions (using 40 uniformly spaced sampling points per cell), the $L^{2}$ and $L^{\infty}$ errors and order of accuracy are listed in Table 7. Again, the errors are second order for the central LDG scheme (1.11)-(1.14).

In principle similar analysis can be performed for higher order polynomials in the central LDG scheme (1.11)(1.14), however the algebra becomes prohibitively complicated. 
TABLE 7. $L^{2}$ and $L^{\infty}$ errors, measured as finite element solutions, and orders of accuracy of the local LDG method (1.11)-(1.14) for $k=1$.

\begin{tabular}{|c|c|c|c|c|}
\hline & \multicolumn{4}{|c|}{$u_{h}$} \\
\hline$h$ & $L^{2}$ error & order & $L^{\infty}$ error & order \\
\hline $2 \pi / 20$ & $4.52 \mathrm{E}-06$ & - & $1.02 \mathrm{E}-06$ & - \\
\hline $2 \pi / 40$ & $1.29 \mathrm{E}-06$ & 1.80 & $2.93 \mathrm{E}-07$ & 1.81 \\
\hline $2 \pi / 80$ & $3.35 \mathrm{E}-07$ & 1.95 & $7.58 \mathrm{E}-08$ & 1.95 \\
\hline $2 \pi / 160$ & $8.45 \mathrm{E}-08$ & 1.99 & $1.91 \mathrm{E}-08$ & 1.99 \\
\hline $2 \pi / 320$ & $2.12 \mathrm{E}-08$ & 2.00 & $4.79 \mathrm{E}-09$ & 2.00 \\
\hline $2 \pi / 640$ & $5.30 \mathrm{E}-09$ & 2.00 & $1.20 \mathrm{E}-09$ & 2.00 \\
\hline
\end{tabular}

\section{A COMPARISON AMONG THE TRADitional LDG SCHEME AND THE TWO VERsions OF CENTRAL LDG METHODS ON OVERLAPPING CELLS}

In this section, we give a quantitative comparison among the traditional LDG scheme and the two versions of central LDG methods on overlapping uniform and non-uniform meshes for solving the heat equation (1.2) with smooth and non-smooth initial conditions respectively. We should keep in mind that the traditional LDG scheme and the first version of the central LDG scheme (1.7)-(1.10) involve the same number of operation counts, when implemented in the matrix form (2.33), however the second version of the central LDG scheme (1.11)-(1.14) is about three times as expensive when implemented in the matrix form (3.24) when noticing the zeros in some of the matrices.

\subsection{The three methods on uniform grids for the heat equation with smooth initial condition}

In [10], results similar to those in Sections 2.3 and 3.3 were obtained for the traditional LDG scheme (1.4), (1.5) applied to the diffusion equation (1.2). For the piecewise constant $k=0$ case, the result for the traditional LDG scheme is the same as the LDG method on overlapping cells (2.32). For the piecewise linear $k=1$ case, it is

$$
\operatorname{Im}\left\{u_{j-\frac{1}{4}}(t)\right\}=\mathrm{e}^{-t} \sin \left(x_{j-\frac{1}{4}}\right)-\frac{\mathrm{e}^{-t} \sin \left(x_{j-\frac{1}{4}}\right)}{24} h^{2}+O\left(h^{3}\right) .
$$

We now compute the traditional LDG scheme (1.4), (1.5) for $k=1$ to (1.2) with $u(x, 0)=\sin (x)$ as the initial condition and with periodic boundary conditions, up to $t=4 \pi$. We use a second order Runge-Kutta method and take a small time step $\Delta t=0.01 h^{2}$. The discrete $L^{2}$ and $L^{\infty}$ errors and order of accuracy of $u_{h}$ at the points $x_{j-\frac{1}{4}}$ are listed in Table 8 . When measuring as finite element solutions (using 40 uniformly spaced sampling points per cell), the $L^{2}$ and $L^{\infty}$ errors and order of accuracy are also listed in Table 8. Again, the errors are second order.

Based on the results in Sections 2.3, 3.3 and above results, we have the following conclusions. Here, the traditional LDG scheme refers to (1.4), (1.5); the first version of the central LDG scheme on overlapping cells refers to (1.7)-(1.10); and the second version of the central LDG scheme on overlapping cells refers to (1.11)(1.14).

For the piecewise constant $k=0$ case:

1. The semi-discrete versions of the traditional LDG scheme, and the two versions of the central LDG schemes on overlapping cells are all stable.

2. The semi-discrete versions of the traditional LDG and first version of central LDG scheme on overlapping cells are the same, and they are both second order accurate at the cell centers $x_{j}$ and first order when measured as finite element solutions. However, the second version of central LDG scheme on overlapping cells is not consistent. 
TABLE 8. Discrete $L^{2}$ and $L^{\infty}$ errors for $u_{h}$, measured at the points $x_{j-\frac{1}{4}}$ and as finite element solutions, and orders of accuracy of the traditional LDG scheme (1.4), (1.5) for $k=1$.

\begin{tabular}{|c|c|c|c|c|c|c|c|c|}
\hline & \multicolumn{3}{|c|}{ Measured at the points $x_{j-\frac{1}{4}}$} & \multicolumn{3}{c|}{ Measured as finite element solutions } \\
\hline$h$ & $L^{2}$ error & Order & $L^{\infty}$ error & Order & $L^{2}$ error & Order & $L^{\infty}$ error & Order \\
\hline $2 \pi / 20$ & $9.69 \mathrm{E}-09$ & - & $1.36 \mathrm{E}-08$ & - & $6.81 \mathrm{E}-08$ & - & $5.60 \mathrm{E}-08$ & - \\
\hline $2 \pi / 40$ & $2.51 \mathrm{E}-09$ & 1.95 & $3.54 \mathrm{E}-09$ & 1.95 & $1.71 \mathrm{E}-08$ & 2.00 & $1.39 \mathrm{E}-08$ & 2.01 \\
\hline $2 \pi / 80$ & $6.32 \mathrm{E}-10$ & 1.99 & $8.94 \mathrm{E}-10$ & 1.99 & $4.29 \mathrm{E}-09$ & 2.00 & $3.46 \mathrm{E}-09$ & 2.00 \\
\hline $2 \pi / 160$ & $1.58 \mathrm{E}-10$ & 2.00 & $2.24 \mathrm{E}-10$ & 2.00 & $1.07 \mathrm{E}-09$ & 2.00 & $8.64 \mathrm{E}-10$ & 2.00 \\
\hline $2 \pi / 320$ & $3.96 \mathrm{E}-11$ & 2.00 & $5.60 \mathrm{E}-11$ & 2.00 & $2.68 \mathrm{E}-10$ & 2.00 & $2.16 \mathrm{E}-10$ & 2.00 \\
\hline $2 \pi / 640$ & $9.90 \mathrm{E}-12$ & 2.00 & $1.40 \mathrm{E}-11$ & 2.00 & $6.70 \mathrm{E}-11$ & 2.00 & $5.40 \mathrm{E}-11$ & 2.00 \\
\hline
\end{tabular}

TABLE 9. $L^{2}$ and $L^{\infty}$ errors and orders of accuracy of the two versions of the central LDG methods on uniform grids for $k=2$.

\begin{tabular}{|c|c|c|c|c|c|c|c|c|}
\hline & \multicolumn{4}{|c|}{ First version } & \multicolumn{4}{c|}{ Second version } \\
\hline$h$ & $L^{2}$ error & Order & $L^{\infty}$ error & Order & $L^{2}$ error & Order & $L^{\infty}$ error & Order \\
\hline $2 \pi / 20$ & $3.70 \mathrm{E}-4$ & - & $3.74 \mathrm{E}-4$ & - & $3.62 \mathrm{E}-4$ & - & $3.36 \mathrm{E}-04$ & - \\
\hline $2 \pi / 40$ & $4.84 \mathrm{E}-5$ & 2.94 & $5.10 \mathrm{E}-5$ & 2.88 & $4.52 \mathrm{E}-5$ & 3.00 & $4.18 \mathrm{E}-5$ & 3.01 \\
\hline $2 \pi / 80$ & $6.49 \mathrm{E}-6$ & 2.90 & $6.99 \mathrm{E}-6$ & 2.88 & $5.66 \mathrm{E}-6$ & 3.00 & $5.22 \mathrm{E}-6$ & 3.00 \\
\hline $2 \pi / 160$ & $8.19 \mathrm{E}-7$ & 2.99 & $8.82 \mathrm{E}-7$ & 2.99 & $7.07 \mathrm{E}-7$ & 3.00 & $6.53 \mathrm{E}-7$ & 3.00 \\
\hline $2 \pi / 320$ & $1.02 \mathrm{E}-7$ & 3.00 & $1.10 \mathrm{E}-7$ & 3.00 & $8.84 \mathrm{E}-8$ & 3.00 & $8.17 \mathrm{E}-8$ & 3.00 \\
\hline $2 \pi / 640$ & $1.28 \mathrm{E}-8$ & 3.00 & $1.38 \mathrm{E}-8$ & 3.00 & $1.10 \mathrm{E}-8$ & 3.00 & $1.02 \mathrm{E}-8$ & 3.00 \\
\hline
\end{tabular}

For the piecewise linear $k=1$ case we have the following conclusions:

1. The semi-discrete versions of the traditional LDG scheme and the two versions of central LDG schemes on overlapping cells are all consistent and stable.

2. Both the traditional LDG scheme and the second version of central LDG scheme on overlapping cells are second order accurate. Comparing (3.25) with (4.1), we can see that the leading term of the error for the second version of the central LDG scheme is about half of that for the traditional LDG scheme for $a=1$. However, the first version of the central LDG scheme on overlapping cells is first order accurate.

For the $k=2$ and $k=3$ cases, it is difficult to make the quantitative analysis as in Sections 2.3 and 3.3. We therefore perform numerical tests to draw some general conclusions. We compute the two versions of the central LDG schemes on overlapping cells and traditional LDG scheme to (1.2) with periodic boundary conditions, up to $t=4 \pi$, with the initial condition $u(x, 0)=\sin (0.01 x)$. For the first version of the central LDG on overlapping cells and the traditional LDG scheme, we use the second order Runge-Kutta method with a small time step $\Delta t=0.001 h^{2}$ to reduce the effect from the time discretization. For the second version of the central LDG on overlapping cells, we use $\tau_{\max }=0.01 h^{2}$, and the second order Runge-Kutta method with a small time step $\Delta t=0.1 \tau_{\max }$ to reduce the effect from the time discretization. The $L^{2}$ and $L^{\infty}$ errors and order of accuracy of these two central LDG schemes are listed in Table 9 for $k=2$ and Table 10 for $k=3$ respectively. The results of the traditional LDG scheme are shown in Table 11 for $k=2$ and $k=3$.

For the $k=4$ cases, we compute the two versions of the central LDG schemes on overlapping cells and traditional LDG scheme to (1.2) with periodic boundary conditions, up to $t=4 \pi$, with the initial condition $u(x, 0)=\sin (0.0001 x)$. For the first version of the central LDG on overlapping cells and the traditional LDG scheme, we use the second order Runge-Kutta method with a small time step $\Delta t=0.0001 h^{2}$ to reduce the effect from the time discretization. For the second version of the central LDG on overlapping cells, we use $\tau_{\max }=0.001 h^{2}$, and the second order Runge-Kutta method with a small time step $\Delta t=0.1 \tau_{\max }$ to reduce 
TABLE 10. $L^{2}$ and $L^{\infty}$ errors and orders of accuracy of the two versions of the central LDG methods on uniform grids for $k=3$.

\begin{tabular}{|c|c|c|c|c|c|c|c|c|}
\hline & \multicolumn{4}{|c|}{ First version } & \multicolumn{5}{c|}{ Second version } \\
\hline$h$ & $L^{2}$ error & Order & $L^{\infty}$ error & Order & $L^{2}$ error & Order & $L^{\infty}$ error & Order \\
\hline $2 \pi / 20$ & $7.31 \mathrm{E}-6$ & - & $9.47 \mathrm{E}-6$ & - & $7.21 \mathrm{E}-6$ & - & $8.19 \mathrm{E}-6$ & - \\
\hline $2 \pi / 40$ & $4.51 \mathrm{E}-7$ & 4.02 & $5.16 \mathrm{E}-7$ & 4.20 & $4.59 \mathrm{E}-7$ & 3.97 & $4.69 \mathrm{E}-7$ & 4.13 \\
\hline $2 \pi / 80$ & $2.84 \mathrm{E}-8$ & 4.00 & $3.05 \mathrm{E}-8$ & 4.08 & $2.87 \mathrm{E}-8$ & 4.00 & $2.93 \mathrm{E}-8$ & 4.00 \\
\hline $2 \pi / 160$ & $1.77 \mathrm{E}-9$ & 4.00 & $1.91 \mathrm{E}-9$ & 4.00 & $1.79 \mathrm{E}-9$ & 4.00 & $1.83 \mathrm{E}-9$ & 4.00 \\
\hline $2 \pi / 320$ & $1.11 \mathrm{E}-10$ & 4.00 & $1.19 \mathrm{E}-10$ & 4.00 & $1.12 \mathrm{E}-10$ & 4.00 & $1.15 \mathrm{E}-10$ & 4.00 \\
\hline $2 \pi / 640$ & $6.92 \mathrm{E}-12$ & 4.00 & $7.47 \mathrm{E}-12$ & 4.00 & $6.93 \mathrm{E}-12$ & 4.02 & $7.08 \mathrm{E}-12$ & 4.02 \\
\hline
\end{tabular}

TABLE 11. $L^{2}$ and $L^{\infty}$ errors and orders of accuracy of the traditional LDG methods uniform grids for $k=2$ and $k=3$.

\begin{tabular}{|c|c|c|c|c|c|c|c|c|}
\hline & \multicolumn{5}{|c|}{$k=2$} & \multicolumn{5}{c|}{$k=3$} \\
\hline$h$ & $L^{2}$ error & Order & $L^{\infty}$ error & Order & $L^{2}$ error & Order & $L^{\infty}$ error & Order \\
\hline $2 \pi / 20$ & $4.20 \mathrm{E}-4$ & - & $3.05 \mathrm{E}-4$ & - & $8.37 \mathrm{E}-6$ & - & $9.84 \mathrm{E}-6$ & - \\
\hline $2 \pi / 40$ & $6.17 \mathrm{E}-5$ & 2.75 & $4.61 \mathrm{E}-5$ & 2.73 & $5.97 \mathrm{E}-7$ & 3.81 & $5.22 \mathrm{E}-7$ & 4.24 \\
\hline $2 \pi / 80$ & $8.04 \mathrm{E}-6$ & 2.94 & $6.33 \mathrm{E}-6$ & 2.86 & $3.86 \mathrm{E}-8$ & 3.95 & $3.13 \mathrm{E}-8$ & 4.05 \\
\hline $2 \pi / 160$ & $1.00 \mathrm{E}-6$ & 3.00 & $7.92 \mathrm{E}-7$ & 3.00 & $2.42 \mathrm{E}-9$ & 4.00 & $1.97 \mathrm{E}-9$ & 4.00 \\
\hline $2 \pi / 320$ & $1.26 \mathrm{E}-7$ & 3.00 & $9.91 \mathrm{E}-8$ & 3.00 & $1.51 \mathrm{E}-10$ & 4.00 & $1.23 \mathrm{E}-10$ & 4.00 \\
\hline $2 \pi / 640$ & $1.57 \mathrm{E}-8$ & 3.00 & $1.24 \mathrm{E}-8$ & 3.00 & $9.42 \mathrm{E}-12$ & 4.00 & $7.61 \mathrm{E}-12$ & 4.00 \\
\hline
\end{tabular}

TABLE 12. $L^{2}$ and $L^{\infty}$ errors and orders of accuracy of the two versions of the central LDG methods on uniform grids for $k=4$.

\begin{tabular}{|c|c|c|c|c|c|c|c|c|}
\hline & \multicolumn{4}{|c|}{ First version } & \multicolumn{4}{c|}{ Second version } \\
\hline$h$ & $L^{2}$ error & order & $L^{\infty}$ error & order & $L^{2}$ error & order & $L^{\infty}$ error & order \\
\hline $2 \pi / 20$ & $1.30 \mathrm{E}-7$ & - & $2.41 \mathrm{E}-7$ & - & $1.30 \mathrm{E}-7$ & - & $2.41 \mathrm{E}-7$ & - \\
\hline $2 \pi / 40$ & $4.07 \mathrm{E}-9$ & 5.00 & $7.54 \mathrm{E}-9$ & 5.00 & $4.06 \mathrm{E}-9$ & 5.00 & $7.51 \mathrm{E}-9$ & 5.00 \\
\hline $2 \pi / 80$ & $1.28 \mathrm{E}-10$ & 5.00 & $2.37 \mathrm{E}-10$ & 5.00 & $1.26 \mathrm{E}-10$ & 5.01 & $2.32 \mathrm{E}-10$ & 5.01 \\
\hline $2 \pi / 160$ & $4.05 \mathrm{E}-12$ & 4.98 & $7.56 \mathrm{E}-12$ & 4.97 & $3.87 \mathrm{E}-12$ & 5.03 & $6.99 \mathrm{E}-12$ & 5.05 \\
\hline
\end{tabular}

TABLE 13. $L^{2}$ and $L^{\infty}$ errors and orders of accuracy of the traditional LDG methods on uniform grids for $k=4$.

\begin{tabular}{|c|c|c|c|c|}
\hline & \multicolumn{4}{|c|}{$k=4$} \\
\hline$h$ & $L^{2}$ error & Order & $L^{\infty}$ error & Order \\
\hline $2 \pi / 20$ & $1.30 \mathrm{E}-7$ & - & $2.40 \mathrm{E}-7$ & - \\
\hline $2 \pi / 40$ & $4.06 \mathrm{E}-9$ & 5.00 & $7.49 \mathrm{E}-9$ & 5.01 \\
\hline $2 \pi / 80$ & $1.26 \mathrm{E}-10$ & 5.01 & $2.30 \mathrm{E}-10$ & 5.02 \\
\hline $2 \pi / 160$ & $3.85 \mathrm{E}-12$ & 5.03 & $6.76 \mathrm{E}-12$ & 5.10 \\
\hline
\end{tabular}

the effect from the time discretization. The $L^{2}$ and $L^{\infty}$ errors and order of accuracy of these two central LDG schemes are listed in Table 12 for $k=4$. The results of the traditional LDG scheme are shown in Table 13.

The numerical results suggest that the first version of the central LDG scheme is of sub-optimal first order accuracy for $k=1$ and of optimal $(k+1)$-th order for $k \neq 1$, and the second version of the central LDG scheme 
TABLE 14. $L^{2}$ and $L^{\infty}$ errors and orders of accuracy of the two versions of the central LDG methods on non-uniform grids for $k=0$.

\begin{tabular}{|c|c|c|c|c|cccc|}
\hline & \multicolumn{4}{|c|}{ First version } & \multicolumn{5}{c|}{ Second version } \\
\hline$h$ & $L^{2}$ error & Order & $L^{\infty}$ error & Order & & & & \\
\hline $2 \pi / 20$ & $1.55 \mathrm{E}-04$ & - & $2.52 \mathrm{E}-05$ & - & $1.36 \mathrm{E}-04$ & - & $2.49 \mathrm{E}-05$ & - \\
\hline $2 \pi / 40$ & $1.32 \mathrm{E}-05$ & 3.55 & $2.78 \mathrm{E}-06$ & 3.40 & $2.43 \mathrm{E}-05$ & 2.49 & $6.44 \mathrm{E}-06$ & 1.95 \\
\hline $2 \pi / 80$ & $1.03 \mathrm{E}-06$ & 3.68 & $3.03 \mathrm{E}-07$ & 2.97 & $1.56 \mathrm{E}-06$ & 0.64 & $3.69 \mathrm{E}-07$ & 0.81 \\
\hline $2 \pi / 160$ & $1.57 \mathrm{E}-07$ & 2.71 & $7.75 \mathrm{E}-08$ & 1.96 & $1.56 \mathrm{E}-05$ & 0.00 & $3.49 \mathrm{E}-06$ & 0.00 \\
\hline $2 \pi / 320$ & $7.68 \mathrm{E}-08$ & 1.03 & $3.75 \mathrm{E}-08$ & 1.05 & $1.56 \mathrm{E}-05$ & 0.00 & $3.49 \mathrm{E}-06$ & 0.00 \\
\hline $2 \pi / 640$ & $3.84 \mathrm{E}-08$ & 1.01 & $1.85 \mathrm{E}-08$ & 1.02 & $1.56 \mathrm{E}-05$ & 0.00 & $3.49 \mathrm{E}-06$ & 0.00 \\
\hline
\end{tabular}

TABLE 15. $L^{2}$ and $L^{\infty}$ errors and orders of accuracy of the two versions of the central LDG methods on non-uniform grids for $k=1$.

\begin{tabular}{|c|c|c|c|c|c|c|c|c|}
\hline & \multicolumn{4}{|c|}{ First version } & \multicolumn{4}{c|}{ Second version } \\
\hline$h$ & $L^{2}$ error & Order & $L^{\infty}$ error & Order & $L^{2}$ error & Order & $L^{\infty}$ error & Order \\
\hline $2 \pi / 20$ & $1.20 \mathrm{E}-4$ & - & $4.62 \mathrm{E}-5$ & - & $3.41 \mathrm{E}-5$ & - & $6.37 \mathrm{E}-6$ & - \\
\hline $2 \pi / 40$ & $1.75 \mathrm{E}-4$ & -0.54 & $6.04 \mathrm{E}-5$ & -0.39 & $4.84 \mathrm{E}-6$ & 2.82 & $1.03 \mathrm{E}-6$ & 2.63 \\
\hline $2 \pi / 80$ & $3.18 \mathrm{E}-5$ & 2.48 & $1.09 \mathrm{E}-5$ & 2.47 & $4.60 \mathrm{E}-7$ & 3.39 & $1.26 \mathrm{E}-7$ & 3.03 \\
\hline $2 \pi / 160$ & $1.08 \mathrm{E}-5$ & 1.55 & $3.75 \mathrm{E}-6$ & 1.54 & $8.45 \mathrm{E}-8$ & 2.44 & $1.96 \mathrm{E}-8$ & 2.68 \\
\hline $2 \pi / 320$ & $2.11 \mathrm{E}-6$ & 2.35 & $7.56 \mathrm{E}-7$ & 2.31 & $2.14 \mathrm{E}-8$ & 1.99 & $5.28 \mathrm{E}-9$ & 1.89 \\
\hline $2 \pi / 640$ & $2.23 \mathrm{E}-6$ & 0.00 & $7.77 \mathrm{E}-7$ & 0.00 & $5.31 \mathrm{E}-9$ & 2.01 & $1.28 \mathrm{E}-9$ & 2.05 \\
\hline
\end{tabular}

is of the optimal $(k+1)$-th order for all $k$. Quantitatively, it seems that the errors for the two versions of the central LDG scheme are close and they are slightly smaller than that for the regular LDG scheme for the same mesh size.

\subsection{The three methods on randomly perturbed non-uniform grids for the heat equation with smooth initial condition}

Since the central LDG methods use two overlapping grids and the interplay between them might be important for the convergence, we numerically study the errors on non-uniform meshes in this subsection. The non-uniform meshes in this subsection are obtained by randomly perturbing each cell interface of a uniform mesh by up to $10 \%$.

As before, for the $k=0$ and $k=1$ cases, we compute the two versions of the central LDG schemes on overlapping cells to (1.2) with periodic boundary conditions, up to $t=4 \pi$, with the initial condition $u(x, 0)=\sin (x)$ on non-uniform meshes. Here for the first version of the central LDG on overlapping cells, we use the second order Runge-Kutta method with a small time step $\Delta t=0.001 h^{2}$ to reduce the effect from the time discretization. For the second version of the central LDG on overlapping cells, we use $\tau_{\max }=0.01 h^{2}$, and the second order Runge-Kutta method with a small time step $\Delta t=0.1 \tau_{\max }$ to reduce the effect from the time discretization. The $L^{2}$ and $L^{\infty}$ errors and order of accuracy of these two central LDG schemes are listed in Table 14 for $k=0$ and in Table 15 for $k=1$, respectively. The results of the second version of the central LDG scheme for $k=0$ are expected since the scheme is not consistent. The results of the first version of the central LDG scheme for the $k=1$ case does not provide a clean order pattern, however Figure 1 shows the relationship between the $L^{2}$ errors and the number of cells for this case, with the order of accuracy being 1.92 by a least square fitting.

For the $k=2$ and $k=3$ cases, the two versions of the central LDG schemes on overlapping cells to (1.2) with periodic boundary conditions, up to $t=4 \pi$, with the initial condition $u(x, 0)=\sin (0.01 x)$ on the same 


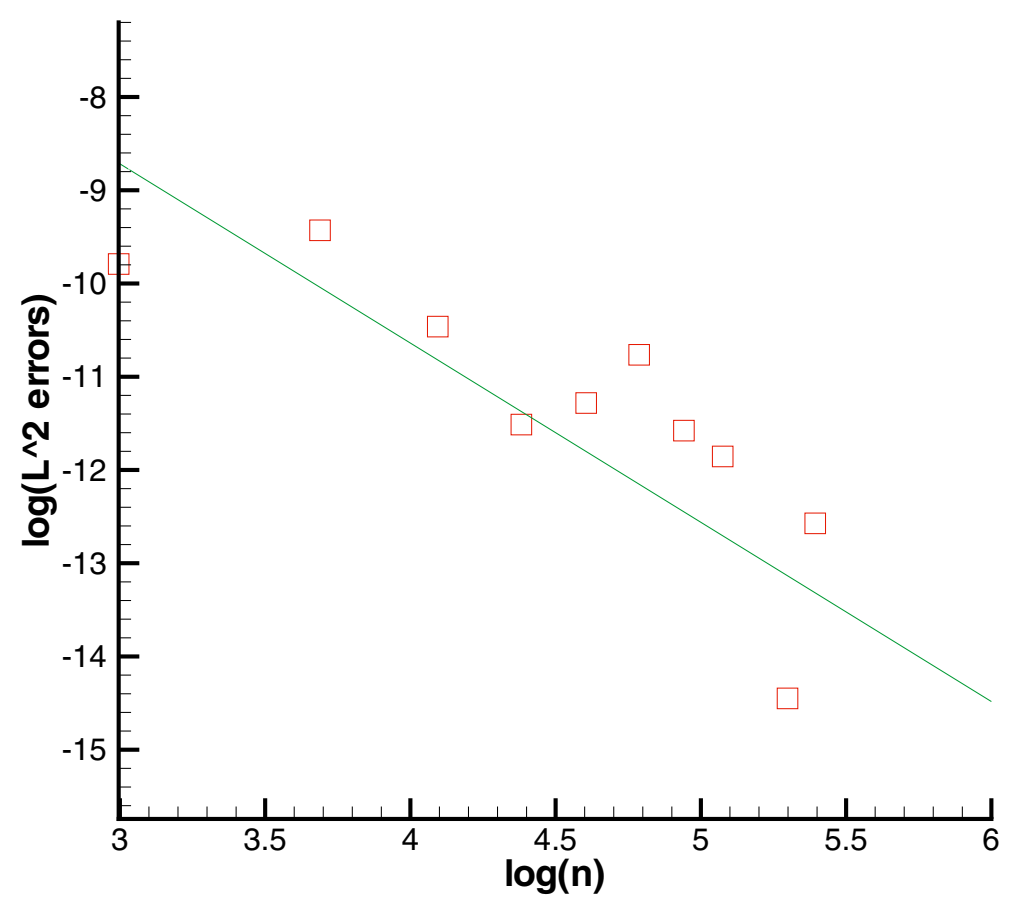

Figure 1. The $L^{2}$ error versus the number of cells, in logarithm scale, obtained by using the first version of the central LDG methods with non-uniform meshes. The symbols (square) are the $L^{2}$ errors and the solid line is the least square fit to the errors.

TABLE 16. $L^{2}$ and $L^{\infty}$ errors and orders of accuracy of the two versions of the central LDG methods on non-uniform grids for $k=2$.

\begin{tabular}{|c|c|c|c|c|c|c|c|c|}
\hline & \multicolumn{4}{|c|}{ First version } & \multicolumn{4}{c|}{ Second version } \\
\hline$h$ & $L^{2}$ error & Order & $L^{\infty}$ error & Order & $L^{2}$ error & Order & $L^{\infty}$ error & Order \\
\hline $2 \pi / 20$ & $3.69 \mathrm{E}-4$ & - & $3.78 \mathrm{E}-4$ & - & $3.62 \mathrm{E}-4$ & - & $3.55 \mathrm{E}-4$ & - \\
\hline $2 \pi / 40$ & $4.86 \mathrm{E}-5$ & 2.93 & $5.28 \mathrm{E}-5$ & 2.84 & $4.55 \mathrm{E}-5$ & 2.99 & $4.48 \mathrm{E}-5$ & 2.99 \\
\hline $2 \pi / 80$ & $6.51 \mathrm{E}-6$ & 2.90 & $7.67 \mathrm{E}-6$ & 2.78 & $5.71 \mathrm{E}-6$ & 3.00 & $5.69 \mathrm{E}-6$ & 2.98 \\
\hline $2 \pi / 160$ & $8.26 \mathrm{E}-7$ & 2.98 & $1.02 \mathrm{E}-6$ & 2.91 & $7.20 \mathrm{E}-7$ & 2.99 & $7.38 \mathrm{E}-7$ & 2.94 \\
\hline $2 \pi / 320$ & $1.03 \mathrm{E}-7$ & 3.00 & $1.33 \mathrm{E}-7$ & 2.93 & $9.01 \mathrm{E}-8$ & 3.00 & $9.23 \mathrm{E}-8$ & 3.00 \\
\hline $2 \pi / 640$ & $1.29 \mathrm{E}-8$ & 3.00 & $1.65 \mathrm{E}-8$ & 3.01 & $1.12 \mathrm{E}-8$ & 3.00 & $1.16 \mathrm{E}-9$ & 2.99 \\
\hline
\end{tabular}

randomly perturbed non-uniform grids are tested. For the first version of the central LDG on overlapping cells, we use the second order Runge-Kutta method with a small time step $\Delta t=0.001 h^{2}$ to reduce the effect from the time discretization. For the second version of the central LDG on overlapping cells, we use $\tau_{\max }=0.01 h^{2}$, and the second order Runge-Kutta method with a small time step $\Delta t=0.1 \tau_{\max }$ to reduce the effect from the time discretization. The $L^{2}$ and $L^{\infty}$ errors and order of accuracy of these two central LDG schemes are listed in Table 16 for $k=2$ and Table 17 for $k=3$, respectively.

For the $k=4$ cases, we compute the two versions of the central LDG schemes on overlapping cells to (1.2) with periodic boundary conditions, up to $t=4 \pi$, with the initial condition $u(x, 0)=\sin (0.0001 x)$ on the same randomly perturbed non-uniform grids. For the first version of the central LDG on overlapping cells, we use the second order Runge-Kutta method with a small time step $\Delta t=0.0001 h^{2}$ to reduce the effect from the time discretization. For the second version of the central LDG on overlapping cells, we use $\tau_{\max }=0.001 h^{2}$, and 
TABLE 17. $L^{2}$ and $L^{\infty}$ errors and orders of accuracy of the two versions of the central LDG methods on non-uniform grids for $k=3$.

\begin{tabular}{|c|c|c|c|c|c|c|c|c|}
\hline & \multicolumn{4}{|c|}{ First version } & \multicolumn{4}{c|}{ Second version } \\
\hline$h$ & $L^{2}$ error & Order & $L^{\infty}$ error & Order & $L^{2}$ error & Order & $L^{\infty}$ error & Order \\
\hline $2 \pi / 20$ & $7.46 \mathrm{E}-6$ & - & $1.06 \mathrm{E}-5$ & - & $7.43 \mathrm{E}-6$ & - & $8.68 \mathrm{E}-6$ & - \\
\hline $2 \pi / 40$ & $4.57 \mathrm{E}-7$ & 4.03 & $6.42 \mathrm{E}-7$ & 4.04 & $4.64 \mathrm{E}-7$ & 4.00 & $5.85 \mathrm{E}-7$ & 3.89 \\
\hline $2 \pi / 80$ & $2.87 \mathrm{E}-8$ & 4.00 & $3.89 \mathrm{E}-8$ & 4.05 & $2.89 \mathrm{E}-8$ & 4.01 & $3.56 \mathrm{E}-8$ & 4.04 \\
\hline $2 \pi / 160$ & $1.80 \mathrm{E}-9$ & 3.99 & $2.55 \mathrm{E}-9$ & 3.93 & $1.81 \mathrm{E}-9$ & 4.00 & $2.31 \mathrm{E}-9$ & 3.95 \\
\hline $2 \pi / 320$ & $1.13 \mathrm{E}-10$ & 4.00 & $1.78 \mathrm{E}-10$ & 3.84 & $1.13 \mathrm{E}-10$ & 4.00 & $1.48 \mathrm{E}-10$ & 3.96 \\
\hline $2 \pi / 640$ & $7.09 \mathrm{E}-12$ & 3.99 & $1.16 \mathrm{E}-11$ & 3.95 & $7.05 \mathrm{E}-12$ & 4.00 & $9.49 \mathrm{E}-12$ & 3.97 \\
\hline
\end{tabular}

TABLE 18. $L^{2}$ and $L^{\infty}$ errors and orders of accuracy of the two versions of the central LDG methods on non-uniform grids for $k=4$.

\begin{tabular}{|c|c|c|c|c|c|c|c|c|}
\hline & \multicolumn{4}{|c|}{ First version } & \multicolumn{4}{c|}{ Second version } \\
\hline$h$ & $L^{2}$ error & Order & $L^{\infty}$ error & Order & $L^{2}$ error & order & $L^{\infty}$ error & Order \\
\hline $2 \pi / 20$ & $1.30 \mathrm{E}-7$ & - & $2.54 \mathrm{E}-7$ & - & $1.30 \mathrm{E}-7$ & - & $2.54 \mathrm{E}-7$ & - \\
\hline $2 \pi / 40$ & $4.12 \mathrm{E}-9$ & 4.98 & $8.04 \mathrm{E}-9$ & 4.98 & $4.11 \mathrm{E}-9$ & 4.98 & $8.01 \mathrm{E}-9$ & 4.99 \\
\hline $2 \pi / 80$ & $1.28 \mathrm{E}-10$ & 5.01 & $2.65 \mathrm{E}-10$ & 4.93 & $1.27 \mathrm{E}-10$ & 5.01 & $2.61 \mathrm{E}-10$ & 4.94 \\
\hline $2 \pi / 160$ & $4.01 \mathrm{E}-12$ & 5.00 & $9.16 \mathrm{E}-12$ & 4.85 & $3.90 \mathrm{E}-12$ & 5.02 & $8.71 \mathrm{E}-12$ & 4.91 \\
\hline $2 \pi / 320$ & $1.26 \mathrm{E}-13$ & 5.00 & $2.80 \mathrm{E}-13$ & 5.03 & $1.14 \mathrm{E}-13$ & 5.09 & $2.30 \mathrm{E}-13$ & 5.24 \\
\hline
\end{tabular}

the second order Runge-Kutta method with a small time step $\Delta t=0.1 \tau_{\max }$ to reduce the effect from the time discretization. The $L^{2}$ and $L^{\infty}$ errors and order of accuracy of these two central LDG schemes are listed in Table 18 for $k=4$.

By comparing the above results, we can see that $L^{2}$ and $L^{\infty}$ errors and order of accuracy of these two central LDG schemes on uniform grids and randomly perturbed non-uniform grids are comparable, with the errors on the non-uniform grids slightly larger for the same number of cells.

\subsection{The three methods on uniform grids for the heat equation with non-smooth initial condition}

In the subsection, we compute the two versions of the central LDG schemes on overlapping cells and the traditional LDG scheme to (1.2) with periodic boundary conditions, up to $t=4 \pi$, with the non-smooth initial condition

$$
u(x, 0)= \begin{cases}1, & |x-\pi| \leq \frac{\pi}{2} \\ 0, & \text { otherwise }\end{cases}
$$

for $k=2$. For the first version of the central LDG methods and the traditional LDG scheme on uniform grids, we use the second order Runge-Kutta method with a small time step $\Delta t=0.0001 h^{2}$ to reduce the effect from the time discretization. The results are shown in Table 19. The order of accuracy is 2 . This is expected as the lack of regularity of the initial condition impacts the order of convergence.

For the second version of the central LDG on overlapping cells, we use $\tau_{\max }=0.1 h^{2}$, and the second order Runge-Kutta method with a small time step $\Delta t=0.01 \tau_{\max }$ to reduce the effect from the time discretization. Table 20 shows the results. The order of accuracy is also 2 . However, the errors are smaller than the traditional LDG or the first version of the central LDG on the same meshes, indicating that the extra numerical dissipation in the second version of the central LDG scheme is helping for non-smooth data. 
TABLE 19. $L^{2}$ and $L^{\infty}$ errors and orders of accuracy of the first version of the central LDG methods and traditional LDG method with non-smooth initial condition for $k=2$.

\begin{tabular}{|c|c|c|c|c|c|c|c|c|}
\hline & \multicolumn{4}{|c|}{ First version } & \multicolumn{4}{c|}{ Traditional LDG } \\
\hline$h$ & $L^{2}$ error & Order & $L^{\infty}$ error & Order & $L^{2}$ error & Order & $L^{\infty}$ error & Order \\
\hline $2 \pi / 20$ & $6.10 \mathrm{E}-8$ & - & $1.37 \mathrm{E}-8$ & - & $6.09 \mathrm{E}-8$ & - & $1.37 \mathrm{E}-8$ & - \\
\hline $2 \pi / 40$ & $1.53 \mathrm{E}-8$ & 2.00 & $3.42 \mathrm{E}-9$ & 2.00 & $1.53 \mathrm{E}-8$ & 2.00 & $3.42 \mathrm{E}-9$ & 2.00 \\
\hline $2 \pi / 80$ & $3.83 \mathrm{E}-9$ & 2.00 & $8.56 \mathrm{E}-10$ & 2.00 & $3.83 \mathrm{E}-9$ & 2.00 & $8.62 \mathrm{E}-10$ & 1.99 \\
\hline $2 \pi / 160$ & $9.64 \mathrm{E}-10$ & 1.99 & $2.18 \mathrm{E}-10$ & 1.98 & $9.67 \mathrm{E}-10$ & 1.99 & $2.38 \mathrm{E}-10$ & 1.86 \\
\hline
\end{tabular}

TABLE 20. $L^{2}$ and $L^{\infty}$ errors and orders of accuracy of the second version of the central LDG methods with non-smooth initial condition for $k=2$.

\begin{tabular}{|c|c|c|c|c|}
\hline & \multicolumn{4}{|c|}{$k=2$} \\
\hline$h$ & $L^{2}$ error & Order & $L^{\infty}$ error & Order \\
\hline $2 \pi / 20$ & $3.07 \mathrm{E}-8$ & - & $6.90 \mathrm{E}-9$ & - \\
\hline $2 \pi / 40$ & $7.66 \mathrm{E}-9$ & 2.00 & $1.72 \mathrm{E}-9$ & 2.01 \\
\hline $2 \pi / 80$ & $1.92 \mathrm{E}-9$ & 2.00 & $4.29 \mathrm{E}-10$ & 2.00 \\
\hline $2 \pi / 160$ & $4.83 \mathrm{E}-10$ & 1.99 & $1.12 \mathrm{E}-10$ & 1.94 \\
\hline
\end{tabular}

\section{Concluding REMARKS}

We have described two versions of the central local discontinuous Galerkin (LDG) methods for solving parabolic equations. Stability analysis and error estimates are given for the linear heat equation. Quantitative analysis and comparisons are given for the traditional LDG scheme and the two versions of central LDG schemes. The central LDG schemes avoid the choice of numerical fluxes and provide duplicative information based on the two overlapping meshes.

\section{REFERENCES}

[1] R. Bellman, The stability of solutions of linear differential equations. Duke Math. J. 10 (1943) 643-647.

[2] P. Ciarlet, The Finite Element Method for Elliptic Problem. North Holland (1975).

[3] B. Cockburn and C.-W. Shu, The local discontinuous Galerkin method for time-dependent convection-diffusion systems. SIAM J. Numer. Anal. 35 (1998) 2440-2463.

[4] B. Cockburn and C.-W. Shu, Runge-Kutta Discontinuous Galerkin methods for convection-dominated problems. J. Sci. Comput. 16 (2001) 173-261.

[5] B. Cockburn, B. Dong, J. Guzman, M. Restelli and R. Sacco, A hybridizable discontinuous Galerkin method for steady-state convection-diffusion-reaction problems. SIAM J. Sci. Comput. 31 (2009) 3827-3846.

[6] Y.J. Liu, C.-W. Shu, E. Tadmor and M. Zhang, Central discontinuous Galerkin methods on overlapping cells with a nonoscillatory hierarchical reconstruction. SIAM J. Numer. Anal. 45 (2007) 2442-2467.

[7] Y.-J. Liu, C.-W. Shu, E. Tadmor and M. Zhang, $L^{2}$ stability analysis of the central discontinuous Galerkin method and a comparison between the central and regular discontinuous Galerkin methods. ESAIM: M2AN 42 (2008) 593-607.

[8] B. van Leer and S. Nomura, Discontinuous Galerkin for diffusion, in Proceedings of 17th AIAA Computational Fluid Dynamics Conference (2005) 2005-5108.

[9] M. van Raalte and B. van Leer, Bilinear forms for the recovery-based discontinuous Galerkin method for diffusion. Comm. Comput. Phys. 5 (2009) 683-693.

[10] M. Zhang and C.-W. Shu, An analysis of three different formulations of the discontinuous Galerkin method for diffusion equations. Math. Models Methods Appl. Sci. 13 (2003) 395-413.

[11] M. Zhang and C.-W. Shu, An analysis of and a comparison between the discontinuous Galerkin and the spectral finite volume methods. Comput. Fluids 34 (2005) 581-592. 